\title{
Approach to combined-function magnets via symplectic slicing
}

\author{
M. Titze* \\ CERN - 6, 1-0015 - CH-1211 Geneva 23, Switzerland \\ and Humboldt-University of Berlin, Unter den Linden 6, D-10099 Berlin, Germany
}

(Received 8 February 2016; published 26 May 2016)

\begin{abstract}
In this article we describe how to obtain symplectic "slice" maps for combined-function magnets, by using a method of generating functions. A feature of this method is that one can use an unexpanded and unsplit Hamiltonian. From such a slice map we obtain a first-order map which is symplectic at the closed orbit. We also obtain a symplectic kick map. Both results were implemented into the widely used program MAD-X to regain, in particular, the twiss parameters for the sliced model of the Proton Synchrotron at CERN. In addition, we obtain recursion equations for symplectic maps of general time-dependent Hamiltonians, which might be useful even beyond the scope of accelerator physics.
\end{abstract}

DOI: 10.1103/PhysRevAccelBeams.19.054002

\section{INTRODUCTION}

Combined-function magnets (CFMs) are magnetic structures consisting of the superposition of a bending magnet with multipoles of higher order. Hereby, the higher-order multipoles follow the curved reference orbit, which is given by the field strength of the bending magnet and the nominal energy. CFMs are used, for example, in the Proton Synchrotron (PS) at CERN for the purpose to save space for other instruments along the ring.

In order to track particles over reasonable long periods in a storage ring, numeric stability must be guaranteed: For example, a period of a few seconds in the PS corresponds to over one million of revolutions. In every revolution, the particles are passing a large number of CFMs and errors in the simulation may sum up dramatically.

The idea of symplectic tracking is that, roughly speaking, instead of attempting to solve the exact equations of motion by an approximation, which introduces errors in the phase space path, one solves exactly (and locally) an approximated equation of motion. This avoids the problem of self-enhanced errors during many repetitions and, instead, introduces systematic errors which are independent on the number of steps.

A common way of how this can be achieved is by slicing the ring into small pieces, in which every step constitute a small symplectic operation. In this article we describe, in an explicit fashion, a possible way of slicing a CFM.

Let us briefly outline the structure of this article: In Subsection II A we follow Ref. [1] and construct the vector potential of general CFMs (excluding solenoids), so that

*malte.titze@cern.ch

Published by the American Physical Society under the terms of the Creative Commons Attribution 3.0 License. Further distribution of this work must maintain attribution to the author(s) and the published article's title, journal citation, and DOI. they satisfy the vacuum Maxwell-equations. Hereby we consider only the interior of these magnets and no fringe fields. Using the technique of expansion series for the vector potential, we explicitly show how the gauge freedom is fixed by the constraints on the shape of the field: We are led to two comparable simple recursion equations which determine the potential uniquely up to any order. Alternative techniques can be found in Refs. [1-3]. In Ref. [4] such an expansion was used in order to determine the symplectic Lie-transformation maps for CFMs.

The aim of this work is to complement this picture by providing particular symplectic maps which describe the transport of coordinates through a small section of a CFM. For brevity we call these maps "slice maps," since they emerge from a low-order expansion of a generating function (GF) with respect to the longitudinal coordinate, and to distinguish them from plain "kick" maps, which do not affect the transverse coordinates. It will turn out that a first-order expansion of such a slice map is suitable for computing chromaticities in a "drift-kick" scenario for CFMs. We finally arrived at a PYTHON code to construct the vector potential for any order in the transverse coordinates and any multipole order and to automatically obtain FORTRAN code of the corresponding first-order slice maps ready for inclusion into the program MAD-X [5].

Next, we will recall in Subsec. II B the construction of the Hamiltonian which describes, in comoving coordinates, the motion of the particle through the CFM. This is meant as an introduction to necessary formulas used later on, and similar to what was described in Refs. [6,7].

In Subsec. II C we turn our attention to the question of how to obtain symplectic transformation maps for general Hamiltonians: We follow the idea of expanding a GF by its time parameter, a concept already formulated in two articles in 1983 by Channell and Ruth respectively [8,9]. Ruth considered the case of Hamiltonians which split into a kinetic- and a potential part, while Channell described the 
method for general Hamiltonians. Later on, Channel and Scovel presented an explicit expansion of a series for timeindependent Hamiltonians [10]. Such an expansion was also described, using a different approach, by Feng and Qin [11].

Here we derive a set of recursion equations describing this series for general Hamiltonians (which might be timedependent) by using a particular type of GFs. By going this way, we do not require further comparison of orders and obtain an algorithm for the coefficients which can straightforwardly be implemented. We will see how the coefficients are generated by the partial derivatives of the Hamiltonian. As usual by such GF techniques, we are led to a system of two families of equations, where one of them is of implicit nature, and by construction, the map is symplectic in any order. A PYTHON demonstration code has been written to apply our result to specific Hamiltonians, for example, a Hamiltonian describing a driven 1D KVenvelope equation, and make some basic tests [5].

After these general considerations we patch all things together and construct symplectic slice maps in Sec. III: We derive a map which emerges from the full unexpanded Hamiltonian by using a first-order expansion of the GF in Subsec. III A. In Subsec. III B we apply a splitting method to obtain a symplectic map for a pure kick, which we have used for tracking, while in Subsec. III C we provide a firstorder map which is symplectic at the origin and which we have used for twiss calculations.

In Sec. IV we discuss in more detail how we implemented the formulas into MAD-X; we provide the explicit formula of the vector potential we have used (up to sextupole terms) and perform some numeric tests: We verify that the chromaticities in the PS after the slicing process are now converging to the same values obtained by Lie-transformation techniques using the Polymorphic Tracking Code (PTC), which is a symplectic integrator for tracking purposes developed by Forest [1]. Furthermore we track an off-momentum particle around several turns through the PS. The tracking results confirm a correct (symplectic) implementation.

\section{PRELIMINARIES}

\section{A. Constructing the vector potential}

Let us begin by explicitly constructing the vector potential of the interior of a CFM, excluding the special case of solenoids, by using a technique of expansion series similar to what can be found in Refs. [1,2]. Let $\vec{A}$ denote a vector potential of the magnetic field $\vec{B}$ in Serret-Frenet coordinates $(x, y, s)$ without torsion. For a detailed treatment of these coordinates we refer the reader to the Appendix. The coordinates $x$ and $y$ denote the transverse part; the coordinate $s$ the longitudinal part with respect to arc-length parametrization. For a general vector field $\vec{X}$ we write $\vec{X}=X_{x} \vec{n}+X_{y} \vec{b}+X_{t} \vec{t}$, where $\vec{t}, \vec{n}$ and $\vec{b}$ are orthonormal, smoothly dependent on $s$, and span the comoving Serret-Frenet system. We will call the $x$ - and $y$-components horizontal and vertical respectively.

With the curvature terms $K_{x}$ and $K_{y}$, the metric $d l^{2}$ in the above coordinate system reads

$$
d l^{2}=\left(1+K_{x} x+K_{y} y\right)^{2} d s^{2}+d x^{2}+d y^{2},
$$

and $\vec{B}=\vec{\nabla} \times \vec{A}$ is given by

$$
\begin{aligned}
B_{t}= & (\vec{\nabla} \times \vec{A})_{t}=\partial_{x} A_{y}-\partial_{y} A_{x}, \\
B_{x}= & (\vec{\nabla} \times \vec{A})_{x}=\frac{1}{1+K_{x} x+K_{y} y} \\
& \times\left[\partial_{y}\left(\left(1+K_{x} x+K_{y} y\right) A_{t}\right)-\partial_{s} A_{y}\right], \\
B_{y}= & (\vec{\nabla} \times \vec{A})_{y}=\frac{1}{1+K_{x} x+K_{y} y} \\
& \times\left[\partial_{s} A_{x}-\partial_{x}\left(\left(1+K_{x} x+K_{y} y\right) A_{t}\right)\right] .
\end{aligned}
$$

Since we are not discussing fringe fields or solenoids, we can assume that $B_{t}=0$ holds inside the CFM, and that $B_{x}$ and $B_{y}$ are independent of $s$. This condition is satisfied, if we set $A_{x}=0=A_{y}$ and $A_{t}, K_{x}$ and $K_{y}$ to be independent of $s$, from which it follows that we are in the situation of Lorenz-gauge $\vec{\nabla} \vec{A}=0$. We obtain

$$
\begin{aligned}
& B_{x}=\frac{1}{1+K_{x} x+K_{y} y} \partial_{y}\left(\left(1+K_{x} x+K_{y} y\right) A_{t}\right), \\
& B_{y}=\frac{-1}{1+K_{x} x+K_{y} y} \partial_{x}\left(\left(1+K_{x} x+K_{y} y\right) A_{t}\right) .
\end{aligned}
$$

Because $\vec{\nabla} \vec{B}=0$ is automatically satisfied, the only requirement on $\vec{A}$ to be Maxwell-conform is the condition $\vec{\nabla} \times \vec{B}=0$ (we consider the vacuum case), which means by Lorenz-gauge that $\Delta \vec{A}=0$ has to be fulfilled. Since the $\vec{B}$ components are independent of $s$ and $B_{t}$ is zero, the only equation which has to be fulfilled is therefore

$$
\begin{aligned}
0= & \partial_{x}\left(\frac{1}{1+K_{x} x+K_{y} y} \partial_{x} G\right) \\
& +\partial_{y}\left(\frac{1}{1+K_{x} x+K_{y} y} \partial_{y} G\right)
\end{aligned}
$$

with $G:=\left(1+K_{x} x+K_{y} y\right) A_{t}$. Equation (2) is equivalent to

$0=\left(1+K_{x} x+K_{y} y\right)\left(\partial_{x}^{2}+\partial_{y}^{2}\right) G-K_{x} \partial_{x} G-K_{y} \partial_{y} G$,

i.e. $\Delta_{\perp} G=-K_{x} B_{y}+K_{y} B_{x}=(\vec{B} \times \vec{K})_{t}$ with $\Delta_{\perp}:=\partial_{x}^{2}+$ $\partial_{y}^{2}$ and $\vec{K}:=\left(K_{x}, K_{y}, 0\right)$. Now let $G=\sum_{k=0}^{\infty} G_{k}$ be a decomposition of $G$ into homogeneous polynomials $G_{k}$ 
of (total) order $k$ in $x$ and $y$. Making a coefficient comparison with respect to $k$, we obtain [1]

$$
\Delta_{\perp} G_{k+1}=\left[-\left(K_{x} x+K_{y} y\right) \Delta_{\perp}+K_{x} \partial_{x}+K_{y} \partial_{y}\right] G_{k} .
$$

Observe that $\Delta_{\perp}=\left(\partial_{x}+i \partial_{y}\right)\left(\partial_{x}-i \partial_{y}\right)$, so if we introduce the quantities $r_{ \pm}:=(x \pm i y) / 2$ and define $\partial_{ \pm}:=\partial_{r_{ \pm}}$, similar as in Ref. [1], the chain rule yields $\partial_{ \pm}=\partial_{x} \mp i \partial_{y}$ and thus $\Delta_{\perp}=\partial_{-} \partial_{+}$. Now according to Lemma B.1 (see Appendix) we can define the quantities $g_{k j} \in \mathbb{C}$ via $G_{k}=$ : $\sum_{j=0}^{k} g_{k j} r_{+}^{k-j} r_{-}^{j}$ and so we get

$$
\begin{aligned}
\Delta_{\perp} G_{k+1}= & -\sum_{j=0}^{k} g_{k j}(k-j) j\left[\bar{\kappa} r_{+}+\kappa r_{-}\right] r_{+}^{k-1-j} r_{-}^{j-1} \\
& +\frac{\kappa}{2} \sum_{j=0}^{k} g_{k j}(k-j) r_{+}^{k-1-j} r_{-}^{j}+\frac{\bar{\kappa}}{2} \sum_{j=0}^{k} g_{k j} j r_{+}^{k-j} r_{-}^{j-1} \\
= & \sum_{j=0}^{k-1}\left[\bar{\kappa} g_{k, j+1}(j+1)(j-k+3 / 2)\right. \\
& \left.+\kappa g_{k j}(k-j)(1 / 2-j)\right] r_{+}^{k-1-j} r_{-}^{j}
\end{aligned}
$$

with $\kappa:=K_{x}+i K_{y}$. On the other hand, the last expression must be equal to

$$
\begin{aligned}
\Delta_{\perp} G_{k+1} & =\sum_{j=0}^{k+1} g_{k+1, j}(k+1-j) j r_{+}^{k-j} r_{-}^{j-1} \\
& =\sum_{j=0}^{k-1} g_{k+1, j+1}(k-j)(j+1) r_{+}^{k-1-j} r_{-}^{j},
\end{aligned}
$$

so for all $0 \leq j \leq k-1$, again by Lemma B.1,

$$
\begin{aligned}
(k-j)(j+1) g_{k+1, j+1}= & \bar{\kappa} g_{k, j+1}(j+1)(j-k+3 / 2) \\
& +\kappa g_{k j}(k-j)(1 / 2-j) .
\end{aligned}
$$

Equation (6) was derived by using the rather general symmetry assumptions above. In order to determine $g_{k, k}$ and $g_{k, 0}$, which span the kernel of $\Delta_{\perp}$ on the space of homogeneous polynomials of degree $k$, and thus completely determine the gauge, we require additional conditions. Note that because $G_{k}=\overline{G_{k}}$, we have $\overline{g_{k j}}=g_{k, k-j}$, so only half of the equations in (6) are necessary and only $g_{k, 0}$ needs to be determined.

Let us assume that $\vec{B}$ has a decomposition into (complex) homogeneous polynomials $w_{k}$ of (total) order $k$ in $x$ and $y$ as follows: $B_{y}+i B_{x}=\sum_{k=0}^{\infty} w_{k}$. Since $\left(1+\bar{\kappa} r_{+}+\kappa r_{-}\right) \times$ $\left(B_{y}+i B_{x}\right)=-\partial_{x} G+i \partial_{y} G=-\partial_{+} G$, this is equivalent to the claim that in order $k \geq 1$ we have

$$
\begin{aligned}
& \sum_{j=0}^{k+1}(k+1-j) g_{k+1, j} r_{+}^{k-j} r_{-}^{j} \\
& \quad=\partial_{+} G_{k+1}=-w_{k}-\left(\bar{\kappa} r_{+}+\kappa r_{-}\right) w_{k-1}
\end{aligned}
$$

and $g_{1,0}=-w_{0}$. Let us assume that $\left.w_{k}\right|_{r_{+}=r_{-}}=\lambda_{k} x^{k}$, where $\lambda_{k} \in \mathbb{C}$ are known; i.e., the expansion of $B_{x}$ and $B_{y}$ are given with respect to the midplane $y=0$. These conditions are fulfilled if and only if, using Eq. (7),

$(k+1) g_{k+1,0}=-\sum_{j=1}^{k}(k+1-j) g_{k+1, j}-2^{k}\left(\lambda_{k}+K_{x} \lambda_{k-1}\right)$.

Equations (6) and (8) completely determine the vector potential $A_{t}$ of the CFM.

Remark A.1.- If we define $w_{k}=: \sum_{j=0}^{k} w_{k j} r_{+}^{k-j} r_{-}^{j}$, then there is a one-to-one map between the sets $\left\{g_{k 0} ; k \geq 1\right\}$ and $\left\{w_{k 0} ; k \geq 0\right\}$ : By Eq. (7) we see immediately that for $k \geq 1$ we have

$$
(k+1) g_{k+1,0}=-w_{k, 0}-\bar{\kappa} w_{k-1,0} .
$$

Conversely, from this equation it follows by induction that

$$
\forall k \geq 0: \sum_{j=0}^{k}(j+1)(-\bar{\kappa})^{k-j} g_{j+1,0}=-w_{k, 0} .
$$

\section{B. The Hamiltonian}

Let us briefly recall the derivation of the Hamiltonian we require. We begin with the well-known Hamiltonian $-p_{s}$ which describes the motion of a particle in a general electromagnetic field in (torsion-free) Serret-Frenet coordinates. This Hamiltonian is derived in the Appendix and can also be found for example in Refs. [6] or [12]; here we are using SI units and the same notation conventions as in Subsec. II A.

$$
\begin{aligned}
p_{s}\left(x, y, t, p_{x}, p_{y},-\mathcal{K} ; s\right) & \\
= & \left(1+K_{x} x+K_{y} y\right)\left[c^{-2}(\mathcal{K}-e \varphi)^{2}-\left(p_{x}-e A_{x}\right)^{2}\right. \\
& \left.-\left(p_{y}-e A_{y}\right)^{2}-m^{2} c^{2}\right]^{1 / 2}+\left(1+K_{x} x+K_{y} y\right) e A_{t} .
\end{aligned}
$$

The parameter $\mathcal{K}$ denotes the total energy of the particle, i.e. with the kinetic momentum $\vec{p}_{\text {kin }}:=\gamma m \vec{v}=\vec{p}-e \vec{A}$ it holds that

$$
\vec{p}_{\text {kin }}^{2}=c^{-2}(\mathcal{K}-e \varphi)^{2}-m^{2} c^{2} .
$$

Let $p:=\left|\vec{p}_{\text {kin }}\right|$ the momentum and $p_{0}$ a corresponding nominal value. As in Ref. [7] we introduce the variable $\hat{\eta}:=\left(p-p_{0}\right) / p_{0}$. Dividing everything by $p_{0}$, we obtain the following (dimensionless) expression 


$$
\begin{aligned}
p_{s} / p_{0}= & \left(1+K_{x} x+K_{y} y\right)\left[(1+\hat{\eta})^{2}-\frac{\left(p_{x}-e A_{x}\right)^{2}}{p_{0}^{2}}\right. \\
& \left.-\frac{\left(p_{y}-e A_{y}\right)^{2}}{p_{0}^{2}}\right]^{1 / 2}+\left(1+K_{x} x+K_{y} y\right) \frac{e}{p_{0}} A_{t} .
\end{aligned}
$$

Let $E_{0}$ be the energy corresponding to the nominal momentum $p_{0}$, i.e. $E_{0}=m \gamma_{0} c^{2}=p_{0} c / \beta_{0}$, and set $\eta:=\left(\mathcal{K}-E_{0}\right) / E_{0}$. Then

$$
\frac{d x}{d s}=\frac{\partial\left(-p_{s}\right)}{\partial p_{x}}=\frac{\partial\left(-p_{s} / p_{0}\right)}{\partial\left(p_{x} / p_{0}\right)}, \quad \frac{d\left(p_{x} / p_{0}\right)}{d s}=-\frac{\partial\left(-p_{s} / p_{0}\right)}{\partial x},
$$

$\frac{d y}{d s}=\frac{\partial\left(-p_{s}\right)}{\partial p_{y}}=\frac{\partial\left(-p_{s} / p_{0}\right)}{\partial\left(p_{y} / p_{0}\right)}, \quad \frac{d\left(p_{y} / p_{0}\right)}{d s}=-\frac{\partial\left(-p_{s} / p_{0}\right)}{\partial y}$,

and

$$
\begin{aligned}
\frac{d(-c t)}{d s} & =-c \frac{\partial\left(-p_{s}\right)}{\partial(-\mathcal{K})}=-c p_{0} \frac{\partial\left(-p_{s} / p_{0}\right)}{\partial\left(\eta / \beta_{0}\right)} \frac{\partial\left(\eta / \beta_{0}\right)}{\partial(-\mathcal{K})} \\
& =\frac{\partial\left(-p_{s} / p_{0}\right)}{\partial\left(\eta / \beta_{0}\right)}, \\
\frac{d\left(\eta / \beta_{0}\right)}{d s} & =\frac{-1}{E_{0} \beta_{0}} \frac{d(-\mathcal{K})}{d s}=\frac{1}{E_{0} \beta_{0}} \frac{\partial\left(-p_{s}\right)}{\partial t}=-\frac{\partial\left(-p_{s} / p_{0}\right)}{\partial(-c t)} .
\end{aligned}
$$

Therefore, $-p_{s} / p_{0}$ corresponds to a Hamiltonian with $x, y$, $-c t, p_{x} / p_{0}, p_{y} / p_{0}$ and $\eta / \beta_{0}=: \bar{\eta}$ as generalized coordinates. Let us denote the normalized momenta $p_{x} / p_{0}$ and $p_{y} / p_{0}$ with $p_{x}$ and $p_{y}$ respectively. The coordinate $-c t$ still changes without limit compared to the other (transverse) coordinates $x$ and $y$. Following Ref. [7], this leads us to the introduction of the variable $\sigma:=s-v_{0} t$, which can be introduced by performing an $s$-dependent canonical transformation, induced by the GF $F$ of type 3:

$$
F\left(\xi, \zeta, \sigma, p_{x}, p_{y}, \bar{\eta} ; s\right):=-p_{x} \xi-p_{y} \zeta+\bar{\eta}(s-\sigma) / \beta_{0} .
$$

We obtain

$$
\begin{aligned}
x & =-\frac{\partial F}{\partial p_{x}}=\xi, \quad p_{\xi}=-\frac{\partial F}{\partial \xi}=p_{x} \\
y & =-\frac{\partial F}{\partial p_{y}}=\zeta, \quad p_{\zeta}=-\frac{\partial F}{\partial \zeta}=p_{y}, \\
-c t & =-\frac{\partial F}{\partial \bar{\eta}}=(\sigma-s) / \beta_{0}, \\
p_{\sigma} & =-\frac{\partial F}{\partial \sigma}=\bar{\eta} / \beta_{0}=\eta / \beta_{0}^{2}
\end{aligned}
$$

and so

$$
\begin{aligned}
& \mathcal{H}\left(\xi, \zeta, \sigma, p_{x}, p_{y}, \bar{\eta} / \beta_{0} ; s\right) \\
& \quad+p_{s} / p_{0}\left(\xi, \zeta,(\sigma-s) / \beta_{0}, p_{x}, p_{y}, \bar{\eta} ; s\right) \\
&=\frac{\partial F}{\partial s}\left(\xi, \zeta, \sigma, p_{x}, p_{y}, \bar{\eta} ; s\right)=\bar{\eta} / \beta_{0}
\end{aligned}
$$

for the new Hamiltonian $\mathcal{H}$. The six equations (15a)-(15c) also prove that $F$ is in fact a GF, since they constitute a bijection between the old and the new coordinates for every $s$. Until here, all considerations in this subsection hold for general electromagnetic fields. For our CFM with the special gauge $A_{x}=0=A_{y}$ discussed in Subsection II A, we then arrive at the following Hamiltonian $\mathcal{H}$ with

$$
\begin{aligned}
\mathcal{H}\left(x, y, \sigma, p_{x}, p_{y}, p_{\sigma} ; s\right) & \\
= & p_{\sigma}-\left(1+K_{x} x+K_{y} y\right) \\
& \times\left(\sqrt{(1+\hat{\eta})^{2}-p_{x}^{2}-p_{y}^{2}}+\frac{e}{p_{0}} A_{t}\right) .
\end{aligned}
$$

Since we do not have any electric fields, we set $\varphi=0$. Then $\hat{\eta}$ is related to the coordinate $\eta$ by

$$
\begin{aligned}
(1+\hat{\eta})^{2} & =\frac{p^{2}}{p_{0}^{2}}=\frac{\mathcal{K}^{2}}{c^{2} p_{0}^{2}}-\left(\frac{m c}{p_{0}}\right)^{2} \\
& =\frac{\mathcal{K}^{2}}{\beta_{0}^{2} E_{0}^{2}}-\frac{1}{\beta_{0}^{2} \gamma_{0}^{2}}=\frac{1}{\beta_{0}^{2}}\left[(1+\eta)^{2}-1+\beta_{0}^{2}\right]
\end{aligned}
$$

i.e. $\hat{\eta}$ is only dependent on $p_{\sigma}$, via the velocity $\beta_{0}$ of the reference particle.

The factor $e / p_{0}$ in front of the potential $A_{t}$ can be absorbed in the coefficients $\lambda_{k}$ of the $\vec{B}$-field, which were introduced in Subsec. II A, and therefore we may drop this factor later. In fact, it is already included in the curvatures $K_{x}$ and $K_{y}$ : Because there are no electric fields, let the reference motion with respect to the Serret-Frenet coordinates be produced by a particle having just the constant nominal momentum $p_{0}$ above. Since our frame of reference was parametrized by arc-length, it holds $v_{0}=d s / d t$. The curvature terms are produced under the influence of the magnetic field $\vec{B}=B_{x} \vec{n}+B_{y} \vec{b}$. This reference particle remains at $x=0=y$ by definition, so it will experience only the constant dipole-terms $B_{x, 0}, B_{y, 0}$. We have $d^{2} \vec{r} / d t^{2}=v_{0}^{2} \overrightarrow{d t} / d s=-v_{0}^{2}\left(K_{x} \vec{n}+K_{y} \vec{b}\right)$ and therefore

$$
\begin{aligned}
-\gamma_{0} m v_{0}^{2}\left(K_{x} \vec{n}+K_{y} \vec{b}\right) & =\gamma_{0} m \frac{d \vec{r}^{2}}{d t^{2}}=e v_{0} \vec{t} \times \vec{B}_{0} \\
& =e v_{0}\left(B_{x, 0} \vec{b}-B_{y, 0} \vec{n}\right)
\end{aligned}
$$

so we obtain 


$$
\begin{aligned}
K_{x} & =\frac{e}{p_{0}} B_{y, 0}, \\
K_{y} & =-\frac{e}{p_{0}} B_{x, 0} .
\end{aligned}
$$

Note that for the reference particle, $\vec{v}_{0} \perp \vec{B}$, so we have $\gamma_{0} m v_{0}^{2} / \rho=e v_{0}\left|\vec{B}_{0}\right|$, thus $e / p_{0}=1 /(B \rho)$, where $\rho$ is the radius of curvature and $B^{2}=B_{x, 0}^{2}+B_{y, 0}^{2}$.

\section{General considerations}

Let $\mathcal{H}$ be a Hamiltonian dependent on the generalized canonical variables $q$ and $p$ and a parameter $s$. Denote by $\Delta s=s^{f}-s$ a small section of the parameter space we are considering, where $s^{f}$ denotes the "final" position. In order to obtain a symplectic map from the initial coordinates at $s$ to the final ones, we assume the existence of an $s$-dependent GF $F$ of type 2 to a Hamiltonian which is zero, i.e. we provide a transformation to a complete set of cyclic coordinates, identify the cyclic coordinates with the final coordinates and attempt to represent them in terms of the initial coordinates. The Hamilton-Jacobi equations for $F$ are

$$
\bar{q}=\partial_{\bar{p}} F, \quad p=\partial_{q} F
$$

As a first necessary condition for such an $F$ we have

$$
\partial_{s} F(q, \bar{p} ; s)=-\mathcal{H}(q, p ; s)
$$

Note the important fact that in this equation the variables $p$ are considered to be functions of $q, \bar{p}$ and $s$. To emphasize this fact, let $\psi(q, \bar{p} ; s):=(q, p ; s)$, so Eq. (20) can be written in the coordinate-independent form as

$$
\partial_{s} F=-\mathcal{H} \circ \psi
$$

Lemma C.1: Let $G$ be a differentiable function of $(q, p ; s)$ and assume that there exist a GF $F$ of type 2 , providing a transformation into a complete set of cyclic coordinates. Then

$$
\begin{aligned}
& \partial_{q^{i}}(G \circ \psi)=\left[\partial_{q^{i}} G+\left(\partial_{p^{j}} G\right) \cdot\left(\partial_{q^{i}} p^{j} \circ \psi^{-1}\right)\right] \circ \psi, \\
& \partial_{\bar{p}^{i}}(G \circ \psi)=\left[\left(\partial_{p^{j}} G\right) \cdot\left(\partial_{\bar{p}^{i}} p^{j} \circ \psi^{-1}\right)\right] \circ \psi,
\end{aligned}
$$

Proof.

$$
\begin{aligned}
\partial_{q^{i}}(G \circ \psi)(q, \bar{p} ; s) & =\left(\partial_{q^{j}} G\right)(\psi(q, \bar{p} ; s)) \partial_{q^{i}} q^{j}+\left(\partial_{p^{j}} G\right)(\psi(q, \bar{p} ; s)) \partial_{q^{i}} p^{j}+\left(\partial_{s} G\right)(\psi(q, \bar{p} ; s)) \partial_{q^{i}} S \\
& =\left[\left(\partial_{q^{i}} G\right)+\left(\partial_{p^{j}} G\right) \cdot\left(\partial_{q^{i}} p^{j} \circ \psi^{-1}\right)\right](\psi(q, \bar{p} ; s)) . \\
\partial_{\bar{p}^{i}}(G \circ \psi)(q, \bar{p} ; s) & =\left(\partial_{q^{j}} G\right)(\psi(q, \bar{p} ; s)) \partial_{\bar{p}^{j}} q^{j}+\left(\partial_{p^{j}} G\right)(\psi(q, \bar{p} ; s)) \partial_{\bar{p}^{i}} p^{j}+\left(\partial_{s} G\right)(\psi(q, \bar{p} ; s)) \partial_{\bar{p}^{i}} S \\
& =\left(\partial_{p^{j}} G\right)(\psi(q, \bar{p} ; s)) \partial_{\bar{p}^{i}} p^{j},
\end{aligned}
$$

and

$$
\begin{aligned}
\partial_{s}(G \circ \psi)(q, \bar{p} ; s) & =\left(\partial_{q^{i}} G\right)(\psi(q, \bar{p} ; s)) \underbrace{\partial_{s} q^{i}}_{0}+\left(\partial_{p^{i}} G\right)(\psi(q, \bar{p} ; s)) \underbrace{\partial_{s} p^{i}}_{\partial_{s} \partial_{q^{i}} F \text { with Eq. }(19)}+\left(\partial_{s} G\right)(\psi(q, \bar{p} ; s)) \\
& =-\left(\partial_{p^{i}} G\right)(\psi(q, \bar{p} ; s))\left(\partial_{q^{i}}(\mathcal{H} \circ \psi)\right)(q, \bar{p} ; s)+\left(\partial_{s} G\right)(\psi(q, \bar{p} ; s)) \\
& =\left[-\partial_{p^{i}} G \cdot \partial_{q^{i}} \mathcal{H}-\partial_{p^{i}} G \cdot\left(\partial_{q^{i}} p^{j} \circ \psi^{-1}\right) \cdot \partial_{p^{j}} \mathcal{H}+\partial_{s} G\right](\psi(q, \bar{p} ; s)),
\end{aligned}
$$

where we have used Eq. (21a) to obtain the last equation.

For brevity let us write $S_{i j}:=\partial_{q^{i}} p^{j} \circ \psi^{-1}$. Then Lemma C.1 yields

$$
\text { and } \begin{aligned}
\partial_{s}\left(S_{i j} \circ \psi\right) & =\left[-\partial_{p^{l}} S_{i j} \cdot \partial_{q^{l}} \mathcal{H}-\partial_{p^{l}} S_{i j} \cdot S_{l k} \cdot \partial_{p^{k}} \mathcal{H}+\partial_{s} S_{i j}\right] \circ \psi, \\
& =\partial_{s}\left(\partial_{q^{i}} p^{j}\right)=\partial_{s} \partial_{q^{i}} \partial_{q^{j}} F=-\partial_{q^{i}} \partial_{q^{j}}(\mathcal{H} \circ \psi) \\
& =-\partial_{q^{i}}\left(\left[\partial_{q^{j}} \mathcal{H}+\partial_{p^{l}} \mathcal{H} \cdot S_{j l}\right] \circ \psi\right) \\
& =-\left(\partial_{q^{i}}\left[\partial_{q^{j}} \mathcal{H}+\partial_{p^{l}} \mathcal{H} \cdot S_{j l}\right]+\partial_{p^{k}}\left[\partial_{q^{j}} \mathcal{H}+\partial_{p^{l}} \mathcal{H} \cdot S_{j l}\right] \cdot S_{i k}\right) \circ \psi .
\end{aligned}
$$

Consequently, 


$$
\partial_{s} S_{i j}=-\partial_{q^{i}}\left[\partial_{q^{j}} \mathcal{H}+\partial_{p^{l}} \mathcal{H} \cdot S_{j l}\right]-\partial_{p^{k}}\left[\partial_{q^{j}} \mathcal{H}+\partial_{p^{l}} \mathcal{H} \cdot S_{j l}\right] \cdot S_{i k}+\partial_{p^{l}} S_{i j} \cdot \partial_{q^{l}} \mathcal{H}+\partial_{p^{k}} S_{i j} \cdot S_{k l} \cdot \partial_{p^{l}} \mathcal{H}
$$

This equation shows that the $s$-derivatives of $S_{i j}$ of any order can entirely be expressed by spatial derivatives of itself and of the Hamiltonian. Second, in order to be able to parametrize the cyclic coordinates by the final coordinates, $\left.F\right|_{s^{f}}=: F_{0}$ must provide the identity transformation. This is guaranteed by setting

$$
F_{0}=q \bar{p}
$$

which can be verified by looking at the Hamilton-Jacobi equations for $F_{0}$. Now let us assume that we can write $F$ in form of a Taylor-expansion around $s^{f}$ (with $K \in \mathbb{N}_{0} \cup\{\infty\}$ ) as

$$
F=F_{0}+\sum_{\mu=1}^{K+1} \frac{1}{\mu !}(-\Delta s)^{\mu} \partial_{s}^{\mu} F\left(q, \bar{p} ; s^{f}\right)=F_{0}-\sum_{\mu=0}^{K} \frac{1}{(\mu+1) !}(-\Delta s)^{\mu+1} \partial_{s}^{\mu}(\mathcal{H} \circ \psi)\left(q, \bar{p} ; s^{f}\right) .
$$

By inserting this expansion (23) into the right-hand side of Eq. (19) we obtain

$$
p=\partial_{q} F=\bar{p}-\sum_{\mu=0}^{K} \frac{1}{(\mu+1) !}(-\Delta s)^{\mu+1} \partial_{q} \partial_{s}^{\mu}(\mathcal{H} \circ \psi)\left(q, \bar{p} ; s^{f}\right),
$$

so all derivatives of $\partial_{q^{i}} p^{j}$ with respect to $q$ and $\bar{p}$ vanish at $s=s^{f}$. This implies that $\left.S_{i j}\right|_{s^{f}} \equiv 0$ and all its higher spatial derivatives vanish at $s^{f}$. The derivatives $\partial_{s}^{\mu}(\mathcal{H} \circ \psi)$ in Eq. (23) are given by Eq. (21c), taken at $s^{f}$. Denote for brevity

$$
\mathcal{H} . G:=\partial_{p^{i}} G \cdot \partial_{q^{i}} \mathcal{H}+\partial_{p^{i}} G \cdot S_{i j} \cdot \partial_{p^{j}} \mathcal{H}=\left\langle\partial_{q} \mathcal{H}+S \partial_{p} \mathcal{H}, \partial_{p} G\right\rangle,
$$

where the summation goes over repeated indices. Hence

$$
\partial_{s}^{\mu}(G \circ \psi)=\partial_{s}^{\mu-1}\left(\left(-\mathcal{H} .+\partial_{s}\right) G \circ \psi\right)=\cdots=\left(-\mathcal{H} .+\partial_{s}\right)^{\mu} G \circ \psi,
$$

and therefore we obtain

$$
F(q, \bar{p} ; s)=q \bar{p}-\sum_{\mu=0}^{K} \frac{1}{(\mu+1) !}(-\Delta s)^{\mu+1}\left(\left(-\mathcal{H} .+\partial_{s}\right)^{\mu} \mathcal{H}\right)\left(\psi\left(q, \bar{p} ; s^{f}\right)\right) .
$$

Since $\partial_{q^{i}}(G \circ \psi)\left(q, \bar{p} ; s^{f}\right)=\left(\partial_{q^{i}} G \circ \psi\right)\left(q, \bar{p} ; s^{f}\right)$ and $\partial_{\bar{p}^{i}}(G \circ \psi)\left(q, \bar{p} ; s^{f}\right)=\left(\partial_{p^{j}} G \circ \psi\right)\left(q, \bar{p} ; s^{f}\right) \underbrace{\left.\partial_{\bar{p}^{i}} p^{j}\right|_{s^{f}}}_{\delta_{i j}}$ by Lemma C.1, we obtain the expressions

$$
\begin{aligned}
& \bar{q}=q-\sum_{\mu=0}^{K} \frac{1}{(\mu+1) !}(-\Delta s)^{\mu+1}\left(\partial_{p}\left(-\mathcal{H} .+\partial_{s}\right)^{\mu} \mathcal{H}\right)\left(q, \bar{p} ; s^{f}\right), \\
& \bar{p}=p+\sum_{\mu=0}^{K} \frac{1}{(\mu+1) !}(-\Delta s)^{\mu+1}\left(\partial_{q}\left(-\mathcal{H} .+\partial_{s}\right)^{\mu} \mathcal{H}\right)\left(q, \bar{p} ; s^{f}\right),
\end{aligned}
$$

using that $\psi\left(q, \bar{p} ; s^{f}\right)=\left(q, p\left(q, \bar{p} ; s^{f}\right) ; s^{f}\right)=$ $\left(q, \bar{p} ; s^{f}\right)$. These equations describe implicitly a symplectic transformation for every $K$ : Equation (27b) constitutes an implicit expression for $\bar{p}$. Once its solution is determined, one can insert it into Eq. (27a) to obtain the overall symplectic solution. In Ref. [8] such a series was described and made explicit for the lowest orders.

\section{SYMPLECTIC APPROACH}

\section{A. First-order symplectic slice map}

At this point let us apply the results of Subsec. II C to our particular Hamiltonian (16). Since this Hamiltonian does not depend on $\sigma$, and all derivatives of the $S_{i j}$-especially with respect to $\sigma$-vanish at the final position, it holds in every order 


$$
p_{\sigma}=\bar{p}_{\sigma}
$$

This is expected, because pure magnetic fields cannot change the energy of the particle.

For a pure drift, this equation holds for all momenta: In this case, $\mathcal{H}$ does only depend on the canonical momenta. Let $\mathcal{W}$ be a sufficiently often continuously differentiable function, dependent on $p$ and $s$, and independent on $q$. Then

$$
\partial_{q^{k}}\left(-\mathcal{H} .+\partial_{s}\right) \mathcal{W}=-\partial_{p^{i}} \mathcal{W} \cdot \partial_{q^{k}} S_{i j} \cdot \partial_{p^{j}} \mathcal{H},
$$

and this expression vanishes at $s=s^{f}$ according to our considerations in Subsec. II C. Especially we conclude that $\bar{p}=p$ holds, by taking $\mathcal{W}=\left(-\mathcal{H} .+\partial_{s}\right)^{\mu-1} \mathcal{H}$ for $\mu \geq 1$ inductively.

For the cyclic coordinates $\bar{q}$ in case of a pure drift, the argument is similar but a bit more subtle in this setting of GFs: Let $\mathcal{F}$ be a function of $q, p$ and $s$, and which can be written as a linear combination of terms in which every summand contains at least one factor of the $S_{i j}$ or a partial derivative of these functions with respect to one of the $q^{k}$ or $p^{k}$. Again by our considerations in Subsection II C it should be clear that $\mathcal{F}$ and its partial derivatives with respect to the $q^{k}$ and $p^{k}$ vanish at $s=s^{f}$. By Eq. (22) we see that $\partial_{s} \mathcal{F}$ is of the same form as $\mathcal{F}$, because the spatial Hesse-Matrix $\partial_{q^{i}} \partial_{q^{j}} \mathcal{H}$ in the expression for $\partial_{s} S_{i j}$ vanishes. We conclude that

$$
\partial_{p^{k}}\left(-\mathcal{H} .+\partial_{s}\right) \mathcal{F}=\partial_{p^{k}}\left(-\partial_{p^{i}} \mathcal{F} \cdot S_{i j} \cdot \partial_{p^{j}} \mathcal{H}+\partial_{s} \mathcal{F}\right)
$$

vanishes at $s=s^{f}$. Now observe that $\left(-\mathcal{H} .+\partial_{s}\right) \mathcal{H}=$ $-\mathcal{H} . \mathcal{H}=-\partial_{p^{i}} \mathcal{H} \cdot S_{i j} \cdot \partial_{p^{j}} \mathcal{H}$ is of the form of $\mathcal{F}$. Consequently, $\partial_{p^{k}}\left(-\mathcal{H} .+\partial_{s}\right)^{\mu} \mathcal{H}$ vanishes at $s=s^{f}$ for $\mu \geq 1$. Summarizing these results we obtain in the case of pure drift the explicit equations

$$
\begin{aligned}
& \bar{q}=q+\Delta s \partial_{p} \mathcal{H}(p), \\
& \bar{p}=p .
\end{aligned}
$$

For a general CFM, let us consider Eqs. (27a) and (27b) for the simplest case $K=0$, which corresponds to a first-order expansion of the GF. In Ref. [13] this was discussed for the case of pure bending magnets. Equation (27b) yields for $\bar{p}_{x}$ :

$$
\bar{p}_{x}=p_{x}+\Delta s\left(K_{x} h+\partial_{x} G\right)=u_{x}+\Delta s K_{x} h,
$$

with the abbreviations $h:=\sqrt{(1+\hat{\eta})^{2}-\bar{p}_{x}^{2}-\bar{p}_{y}^{2}}, u_{x}:=p_{x}+$ $\Delta s \partial_{x} G$ and $G=\left(1+K_{x} x+K_{y} y\right) A_{t}$ (see Subsection II A). Similarly we have such an equation for $\bar{p}_{y}$. Then we conclude

$$
\begin{aligned}
h^{2}= & (1+\hat{\eta})^{2}-\bar{p}_{x}^{2}-\bar{p}_{y}^{2} \\
= & (1+\hat{\eta})^{2}-\left(u_{x}+\Delta s K_{x} h\right)^{2}-\left(u_{y}+\Delta s K_{y} h\right)^{2} \\
= & (1+\hat{\eta})^{2}-u_{x}^{2}-u_{y}^{2}-2 \Delta s h\left(u_{x} K_{x}+u_{y} K_{y}\right) \\
& -\Delta s^{2} h^{2}\left(K_{x}^{2}+K_{y}^{2}\right),
\end{aligned}
$$

and therefore obtain the quadratic equation $h^{2}+\xi h+$ $\zeta=0$, with the abbreviations

$\xi:=2 \Delta s \frac{u_{x} K_{x}+u_{y} K_{y}}{1+\Delta s^{2}\left(K_{x}^{2}+K_{y}^{2}\right)}, \quad \zeta:=\frac{u_{x}^{2}+u_{y}^{2}-(1+\hat{\eta})^{2}}{1+\Delta s^{2}\left(K_{x}^{2}+K_{y}^{2}\right)}$.

Consequently, $h=-\xi / 2+\sqrt{\xi^{2} / 4-\zeta}$, where we have determined the sign in front of the square root by the condition that in case $\Delta s=0$ we have $\bar{p}_{x}=p_{x}=u_{x}$ and $\bar{p}_{y}=p_{y}=u_{y}$. The now determined $h$ yields an explicit symplectic map for the momenta when inserted into Eq. (30). For the coordinate $\bar{x}$, Eq. (27a) yields, together with Eq. (30),

$$
\begin{aligned}
\bar{x} & =x+\Delta s\left(1+K_{x} x+K_{y} y\right) \bar{p}_{x} / h \\
& =x+\Delta s\left(1+K_{x} x+K_{y} y\right)\left(u_{x} / h+\Delta s K_{x}\right),
\end{aligned}
$$

and similarly we have such an equation for $\bar{y}$. For $\bar{\sigma}$ we first note that, by implicit differentiation of Eq. (17), we obtain

$$
(1+\hat{\eta}) \frac{\partial \hat{\eta}}{\partial p_{\sigma}}=1+\beta_{0}^{2} p_{\sigma}
$$

thus

$$
\begin{aligned}
\bar{\sigma}= & \sigma+\Delta s \partial_{p_{\sigma}} \mathcal{H}=\sigma+\Delta s \\
& -\Delta s\left(1+K_{x} x+K_{y} y\right)\left(1+\beta_{0}^{2} p_{\sigma}\right) / h .
\end{aligned}
$$

In summary, we obtain the following symplectic map:

$$
\begin{aligned}
& x^{f}=x^{i}+\Delta s\left(1+K_{x} x^{i}+K_{y} y^{i}\right)\left(u_{x}^{i} / h+\Delta s K_{x}\right), \\
& p_{x}^{f}=u_{x}^{i}+\Delta s K_{x} h, \\
& y^{f}=y^{i}+\Delta s\left(1+K_{x} x^{i}+K_{y} y^{i}\right)\left(u_{y}^{i} / h+\Delta s K_{y}\right), \\
& p_{y}^{f}=u_{y}^{i}+\Delta s K_{y} h, \\
& \sigma^{f}=\sigma^{i}+\Delta s-\Delta s\left(1+K_{x} x^{i}+K_{y} y^{i}\right)\left(1+\beta_{0}^{2} p_{\sigma}^{i}\right) / h,
\end{aligned}
$$

$p_{\sigma}^{f}=p_{\sigma}^{i}$,

where $h$ (and $G$ ) are taken at the initial coordinates. Note that $\partial_{x, y} G=\mp\left(1+K_{x} x+K_{y} y\right) B_{y, x}$, which can be 
inserted into these expressions in order to obtain the fielddependency of the transformation.

\section{B. Symplectic kick}

For a "drift-kick" scenario, the slice map (34a)-(34f) is however not used: For example in MAD-X after the slicing process, the tracking through the CFM is performed via consecutive drifts and kicks. To obtain the "kick" part of this procedure, the inverse of a "drift" would have to be applied in either the slice map or the full Lie-transformation map in Ref. [4]. This however would lead to an unnecessary blow up of code. Instead of doing that, we follow a splitting technique described in Ref. [13]. For a discussion of various splitting techniques the reader can consult Ref. [1].

Equation (16) in case of no fields gives the drift part with respect to vacuum

$$
\mathcal{H}_{\text {drift }}\left(p_{x}, p_{y}, p_{\sigma}\right):=p_{\sigma}-\sqrt{(1+\hat{\eta})^{2}-p_{x}^{2}-p_{y}^{2}} .
$$

Now, inside the magnet, this Hamiltonian would change toward the full Hamiltonian $\mathcal{H}$. However, in the case of a drift-kick decomposition, this change is assumed to take place infinitesimally at a certain point $s_{0}$ with a particular strength. At this point, only those parts of $\mathcal{H}^{\prime}:=\mathcal{H}-\mathcal{H}_{\text {drift }}$ which produce a kick in the spatial momenta $p_{x}$ and $p_{y}$ have to be taken into consideration. So what we have to do is to sort out those parts of $\mathcal{H}^{\prime}$ which contain the momenta (since offsets are computed via $\partial \mathcal{H} / \partial p_{x}$ etc.).

Since the physical kicks remain very small with respect to the reference trajectory, we can take a look at the expansion of the square root

$$
\sqrt{(1+\hat{\eta})^{2}-p_{x}^{2}-p_{z}^{2}}=1+\hat{\eta}-\frac{1}{2} \frac{p_{x}^{2}+p_{z}^{2}}{1+\hat{\eta}}+\cdots .
$$

Then we see that

$$
\mathcal{H}_{\text {kick }}^{\prime}:=-\left[\left(K_{x} x+K_{y} y\right)(1+\hat{\eta})+G(x, y)\right] \Delta s \delta\left(s-s_{0}\right)
$$

is the Hamiltonian we are interested in, where we have accounted for the fact that it acts instantaneous by the factor $\Delta s \delta\left(s-s_{0}\right)$. Integration of the corresponding Hamiltonequations for $\mathcal{H}_{\text {kick }}^{\prime}$ yields the following symplectic kick map:

$$
\begin{aligned}
& x^{f}=x^{i}, \\
& p_{x}^{f}=p_{x}^{i}+\Delta s\left(K_{x}(1+\hat{\eta})+\partial_{x} G\right), \\
& y^{f}=y^{i}, \\
& p_{y}^{f}=p_{y}^{i}+\Delta s\left(K_{y}(1+\hat{\eta})+\partial_{y} G\right),
\end{aligned}
$$

$$
\begin{aligned}
& \sigma^{f}=\sigma^{i}-\Delta s\left(K_{x} x+K_{y} y\right)\left(1+\beta_{0}^{2} p_{\sigma}^{i}\right) /(1+\hat{\eta}), \\
& p_{\sigma}^{f}=p_{\sigma}^{i} .
\end{aligned}
$$

\section{First-order drift-kick map}

The map described in Subsec. III B constitutes a pure kick and can be used for tracking, but for chromaticity calculations it lacks any information about the dependency of the transverse coordinates with respect to the other coordinates. This information is generated during the slicing process by composition of the drifts and kicks, but if it cannot be obtained by other means (like numeric differentiation), we have to use a different approach.

Fortunately, the slice map (34a)-(34f) can be used in order to obtain a map which is first-order in $\Delta s$, contains the necessary information and is symplectic at the origin. Symplecticity at the origin is sufficient for twiss calculations, as long as the reference trajectory inside the CFM can be assumed to be located at zero. Besides of converging to the correct values (see later), the advantage in using such a map in comparison to the possibilities described in Subsec. III B is in particular that it does not lead to a large blow up in code [5].

Let $T$ constitute a slice map according to Eqs. (34a)-(34f) with respect to length $\Delta s:=s^{f}-s_{0}$, in which we drop all higher orders in $\Delta s$. This means that the function $h$ in this transformation is replaced by $h_{0}:=\sqrt{(1+\hat{\eta})^{2}-p_{x}^{2}-p_{y}^{2}}$. Let $s_{0}<s_{1}<s^{f}, \Delta s_{1}:=s_{1}-s_{0}$ and $\Delta s_{2}:=s^{f}-s_{1}$, i.e. $\Delta s=\Delta s_{1}+\Delta s_{2}$. Let $D_{i}$ be drifts with respect to $\Delta s_{i}$ for $i=1,2$. Then we would like to compute the composition $D_{2}^{-1} \circ T \circ D_{1}^{-1}$, since this map constitutes the necessary kick map in a "drift-kick-drift" scenario within $\Delta s$, where the kick appears at $s_{1}$.

Let $T(v)=: v+\Delta s f(v)$, i.e. $f$ is given via Eqs. (34a)(34f) in which $h$ is replaced by $h_{0}$. By Eqs. (29a) and (29b) we have $D_{i}^{-1}(v)=v-\Delta s_{i} f_{0}(v)$, where $f_{0}$ is the map $f$ without any field terms. Using $f(v+\Delta s w)=f(v)+\mathcal{O}(\Delta s)$ we get

$$
\begin{aligned}
D_{2}^{-1} \circ T \circ D_{1}^{-1}(v) & \\
& =D_{2}^{-1} \circ T\left(v-\Delta s_{1} f_{0}(v)\right) \\
& =D_{2}^{-1}\left(v-\Delta s_{1} f_{0}(v)+\Delta s f\left(v-\Delta s_{1} f_{0}(v)\right)\right) \\
& =D_{2}^{-1}(v-\Delta s_{1} f_{0}(v)+\Delta s f(v)+\underbrace{\Delta s \mathcal{O}\left(\Delta s_{1}\right)}_{\mathcal{O}\left(\Delta s^{2}\right)}) \\
& =v-\Delta s f_{0}(v)+\Delta s f(v)+\mathcal{O}\left(\Delta s^{2}\right) .
\end{aligned}
$$

This means that the first-order term of $D_{2}^{-1} \circ T \circ D_{1}^{-1}$ with respect to $\Delta s$ corresponds to $f-f_{0}$. In addition we see that in first-order it does not matter where the kick is located between the two drifts; especially one drift of length $\Delta s$ is sufficient. Using this result, we obtain a drift-kick map in first-order $\Delta s$ : 


$$
\begin{aligned}
& x^{f}=x^{i}+\Delta s\left(K_{x} x^{i}+K_{y} y^{i}\right) p_{x}^{i} / h_{0}, \\
& p_{x}^{f}=p_{x}^{i}+\Delta s\left(K_{x} h_{0}+\partial_{x} G\right), \\
& y^{f}=y^{i}+\Delta s\left(K_{x} x^{i}+K_{y} y^{i}\right) p_{y}^{i} / h_{0}, \\
& p_{y}^{f}=p_{y}^{i}+\Delta s\left(K_{y} h_{0}+\partial_{y} G\right), \\
& \sigma^{f}=\sigma^{i}-\Delta s\left(K_{x} x^{i}+K_{y} y^{i}\right)\left(1+\beta_{0}^{2} p_{\sigma}^{i}\right) / h_{0}, \\
& p_{\sigma}^{f}=p_{\sigma}^{i} .
\end{aligned}
$$

It turns out that this map is symplectic at the design trajectory (the "closed orbit") at zero, but not symplectic in general. However it is well-suited for MAD-X for computation of the twiss parameters, since these parameters require the knowledge of the transport map only around the closed orbit, which we assume to be at zero inside the CFM. The symplecticity at zero is crucial in order to remain in the symplectic group during composition.

We remark that if we would expand the transformation to second order, by first going to second order in (27a) and (27b), which is always a symplectic, and then making a similar procedure as above, by using iterative Taylor-expansions of the derivatives of the Hamiltonian around $\left(q, p ; s^{f}\right)$ in order to make the equations explicit, we would obtain a nonsymplectic transformation, even at zero [5]. This nonsymplecticity introduces artificial errors during composition which will negate any gain one would expect when going to second-order in this fashion.

\section{MAD-X IMPLEMENTATION}

\section{A. Combined-function potential}

The higher-order terms (the order here is taken with respect to the transverse coordinates) of the transformation map can be obtained by deriving the corresponding transformation maps and evaluating them at zero. In MAD-X, the proper factorial has to be taken into account. The higherorder terms $r e$ and te in MAD-X correspond, respectively, to the first- and second coefficients in front of the polynomial expansion of the map around the ideal trajectory at zero.

The field components in MAD-X are given as coefficients $B_{x, k}, B_{y, k}$ (times the normalization $e / p_{0}$ ) for $k=0$, 1 , etc. of the Taylor-expansion around $x=0$

$B_{x}(x, 0)=\sum_{k=0}^{\infty} \frac{1}{k !} B_{x, k} x^{k}, \quad B_{y}(x, 0)=\sum_{k=0}^{\infty} \frac{1}{k !} B_{y, k} x^{k}$,

where the dipole terms $(k=0)$ are related to the curvatures $K_{x}$ and $K_{y}$ according to Eqs. (18a) and (18b). The factorials have to be taken into account when implementing the code, see Appendix E. The possibility of rotated input can be

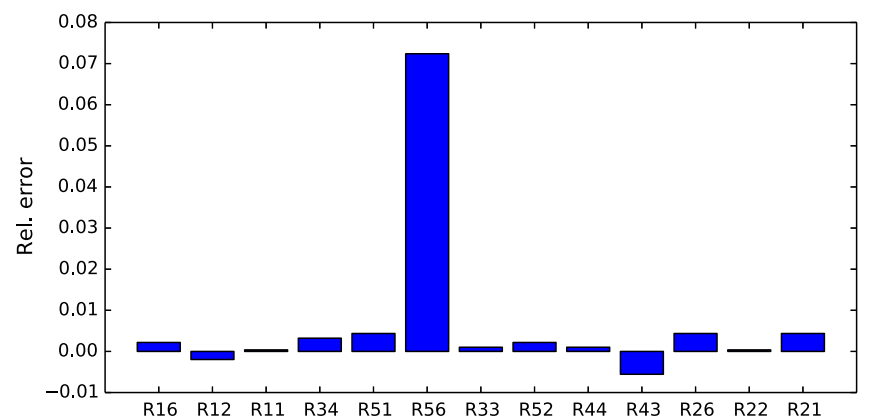

FIG. 1. Relative errors for various re-entries for 4 slices of a single CFM with quadrupole and sextupole component against the Lie-transformation coefficients. Those errors which are zero are not shown.

straightforwardly implemented by changing the coefficients $\lambda_{k}$ accordingly, or making a rotation before- and afterwards. We have included fields up to sextupoles and used an expansion of up to total order 4:

$$
\begin{aligned}
G= & \frac{K_{x}^{2} g}{24} y^{4}-\frac{K_{x}^{2} x^{2}}{2}+\frac{K_{x} K_{y}}{6} g x y^{3}-K_{x} K_{y} x y-\frac{K_{x} g}{3} x^{3} \\
& +\frac{K_{x} g}{2} x y^{2}-\frac{K_{x} h}{8} x^{4}+\frac{K_{x} h}{2} x^{2} y^{2}-\frac{K_{x} h}{24} y^{4} \\
& -K_{x} x-\frac{K_{y}^{2} g}{24} y^{4}-\frac{K_{y}^{2} y^{2}}{2}+\frac{K_{y} g}{6} y^{3}+\frac{K_{y} h}{6} x y^{3} \\
& -K_{y} y-\frac{g x^{2}}{2}+\frac{g y^{2}}{2}-\frac{h x^{3}}{6}+\frac{h x}{2} y^{2},
\end{aligned}
$$

where $g$ denotes the strength of the quadrupole and $h$ the strength of the sextupole. The expression was obtained by an interactive PYTHON script via the SYMPY module [5,14]. This potential yields the desired expansion

$$
\begin{aligned}
& \left.B_{x}\right|_{y=0}=-K_{y}, \\
& \left.B_{y}\right|_{y=0}=K_{x}+g x+\frac{1}{2} h x^{2},
\end{aligned}
$$

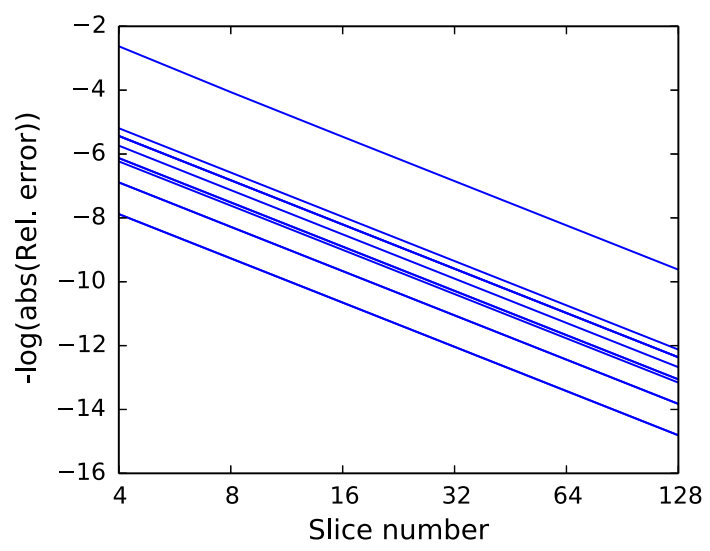

FIG. 2. Counter-check with convergence behavior of the relative errors for the re-coefficients of Fig. 1. 

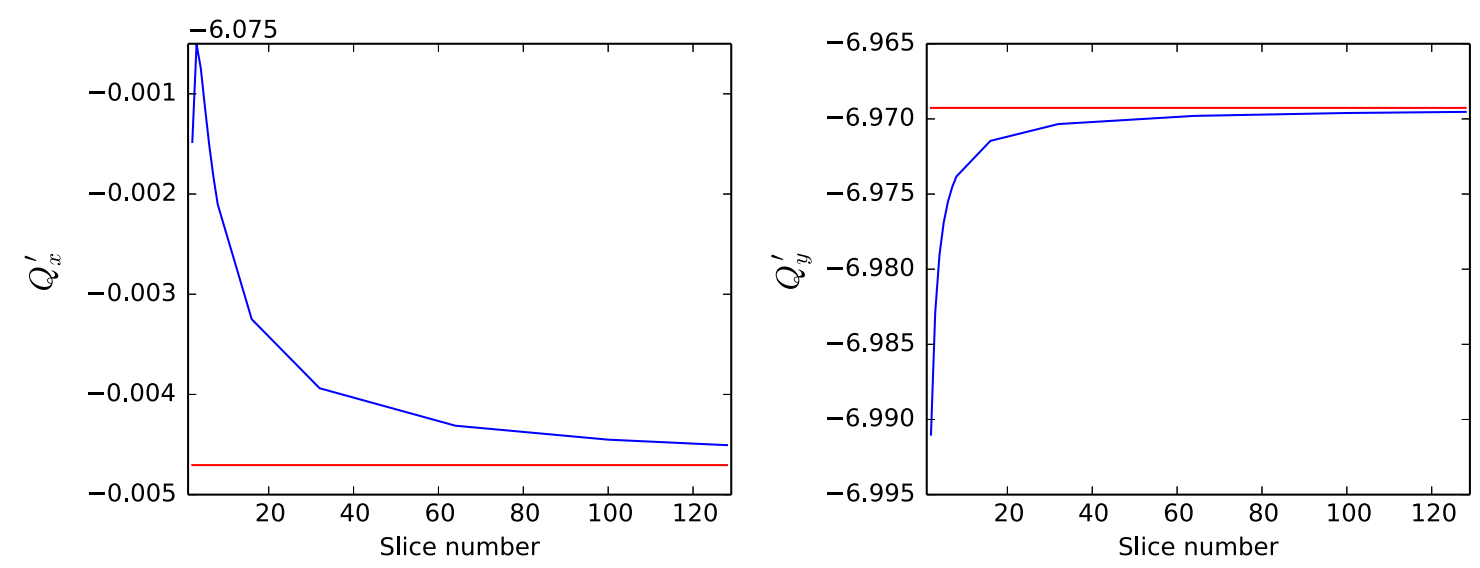

FIG. 3. Convergence behavior of the horizontal- and vertical chromaticities in the PS against the PTC results (red).

as one can verify straightforwardly. Note that the factor $e / p_{0}$ is absorbed in the $K$ 's, as discussed earlier in Subsec. II B. For the details of the necessary code changes in MAD-X, we refer the reader to the Appendix.

\section{B. Numerical tests}

1. We have tested our first-order drift-kick map against the Lie-transformation formulas of Ref. [4] (which are implemented in MAD-X and yield the same results as PTC) for a single CFM. The formulas were implemented in a modified version of MAD-X (v.5.02.07) and compared to the unmodified one. For the details of the code changes, see the Appendix. The magnet was invoked without fringe fields, and the corresponding sectorfiles, which contain the Lie-transformation coefficients, were compared. The firstorder coefficients are denoted by re; the second-order coefficients by te.

As an example, we have listed in Fig. 1 the relative error in the re-entries in case of 4 slices for a CFM against the results of the Lie-transformation. The magnet had a total length of $3.92 \mathrm{~m}$ and bends a $100 \mathrm{GeV}$ proton by an angle of $\pi / 8$. On top of its dipole field, it had a quadrupole and a sextupole field of strengths $k_{1}=-0.03644314869[\mathrm{~m}]^{-2}$ and $k_{2}=-4 \times 10^{-2}[\mathrm{~m}]^{-3}$ respectively. It turns out that the skyline of error bars looks similar for other slice numbers, while the error bar of R56 is somewhat standing out relative to the others: These relative errors, including the ones of the te-entries (not shown), follow an expected linear convergence behaviour in dependency of the slice number, see Fig. 2. All plots in this article were created using the PyLab bundle [15-17].

2. As a second test of the first-order drift-kick map, we have computed the twiss parameters for the PS after thin slicing and obtained chromaticity results listed in Table I. The tunes were at $Q_{x}=6.44987804$ and $Q_{y}=$ 6.46247186. Note that in MAD-X the horizontal chromaticity $Q_{x}^{\prime}$ is defined as $\partial Q_{x} / \partial\left(\beta_{0} p_{\sigma}\right)$ (and correspondingly the vertical one $Q_{y}^{\prime}$ ). For comparison, the result of the unmodified MAD-X v.5.02.07 code gives $Q_{x}^{\prime}=$ -7.33336900 and $Q_{y}^{\prime}=-7.33108397$ (with 128 slices) which is over $20 \%$ off in horizontal and $5 \%$ off in vertical direction from the Lie-transformation reference values at $Q_{x, 0}^{\prime}=-6.079705356$ and $Q_{y, 0}^{\prime}=-6.969268222$. The fringe fields of the bending magnets were again switched off for comparison; otherwise the convergence of the chromaticities towards the PTC values would retain a small offset of about $0.01 \%$ in both planes. The table shows that with just 4 slices we obtain a precision lower than $0.2 \%$. In Fig. 3 the convergence behavior of the chromaticities against the PTC results is shown.

TABLE I. Simulated chromaticity in the PS for various slice numbers.

\begin{tabular}{lcccc}
\hline \hline Slice number & $Q_{x}^{\prime}$ & $Q_{y}^{\prime}$ & $Q_{x}^{\prime} / Q_{x, 0}^{\prime}[\%]$ & $Q_{y}^{\prime} / Q_{y, 0}^{\prime}[\%]$ \\
\hline 128 & -6.07950629 & -6.96953282 & $3.27425407 \times 10^{-3}$ & $3.79662529 \times 10^{-3}$ \\
64 & -6.07931210 & -6.96980020 & $6.46840557 \times 10^{-3}$ & $7.63325478 \times 10^{-3}$ \\
32 & -6.07893833 & -6.97034331 & $1.26162199 \times 10^{-2}$ & $1.54260816 \times 10^{-2}$ \\
16 & -6.07824933 & -6.97146261 & $2.39489566 \times 10^{-2}$ & $3.14866056 \times 10^{-2}$ \\
8 & -6.07710544 & -6.97383113 & $4.27638652 \times 10^{-2}$ & $6.54718954 \times 10^{-2}$ \\
4 & -6.07574514 & -6.97905865 & $6.51383376 \times 10^{-2}$ & $1.40479986 \times 10^{-1}$ \\
2 & -6.07648008 & -6.99100731 & $5.30498077 \times 10^{-2}$ & $3.11927814 \times 10^{-1}$ \\
\hline \hline
\end{tabular}



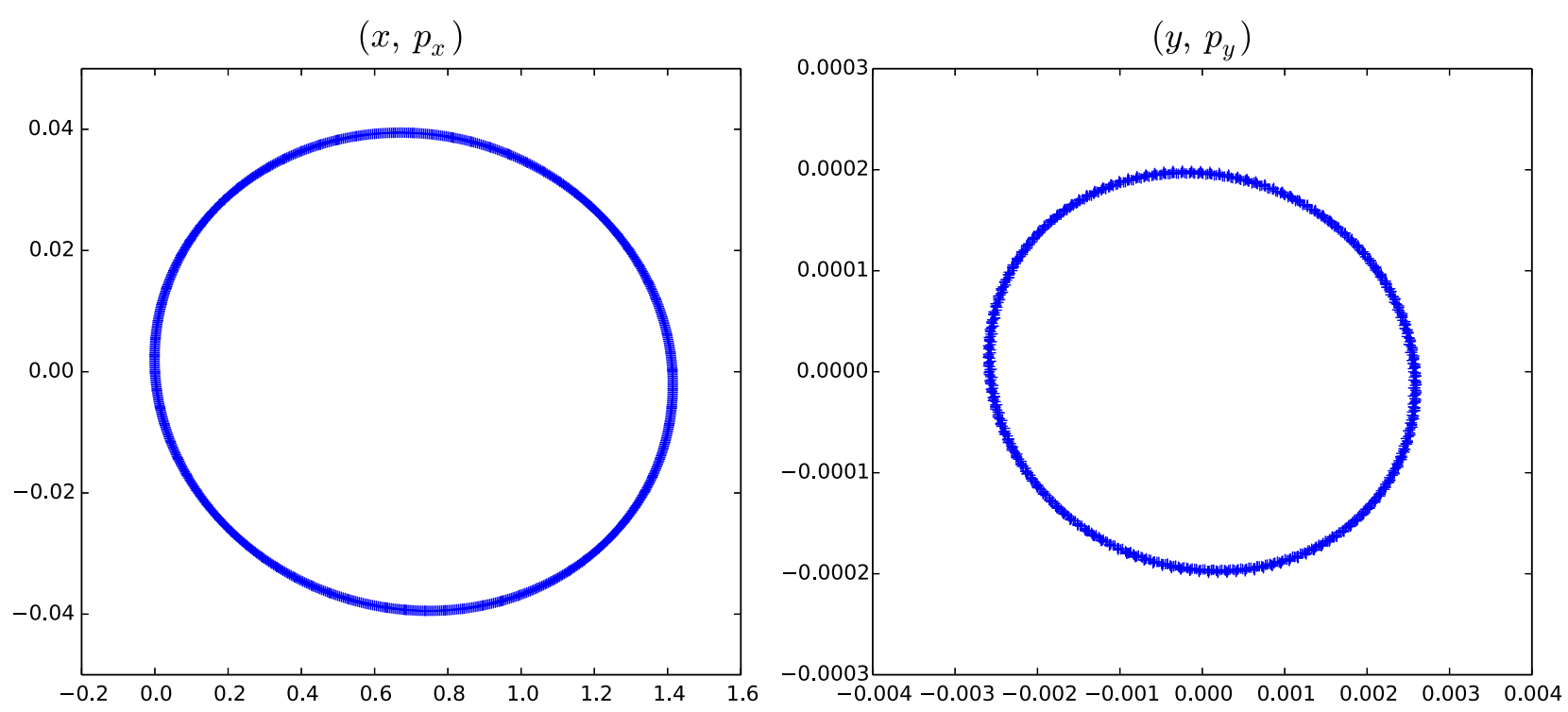

FIG. 4. Horizontal and vertical phase space with the corrected (symplectic) code. The units are [m] and [rad] respectively.

3. To check our symplectic kick map for tracking purposes, described in Subsec. III B, we have simulated 1000 turns of a test particle through the PS, which was given a small offset in its longitudinal momentum. One can clearly see the symplecticity of the new code in Fig. 4. Every CFM was sliced 4 times (and fringe fields were switched on).

\section{CONCLUSIONS}

The methods presented here originated out of the need to obtain correct chromaticities for the PS after a thin-lens slicing of the machine; in particular this is required for those frozen-space-charge models which utilize the thinlens description in the MAD-X tracking code. The resulting values of the modified program now converge toward the Lie-transformation coefficients, while symplecticity is maintained overall. Hereby the map used for computing the twiss parameters is first-order in the longitudinal step length $\Delta s$ and symplectic at the origin, while the kick map used for tracking is symplectic everywhere.

We found that for chromaticity calculations of the entire PS, which depend on the second-order transformation coefficients, a comparable low slice number of around four is already sufficient to correct the error in the horizontal chromaticity towards $0.2 \%$ which was in the order of $20 \%$ for the unmodified code. Moreover, we have corrected the tracking of the old code in case of CFMs.

We remark that a straightforward implementation of the thin-lens kick map by concatenating the second-order thick-lens transformation coefficients with the corresponding inverse drift coefficients would also work in principle, but leads to a large blow-up of code which is undesirable [5]. The method presented here is the most appropriate way we found for a thin-lens implementation of CFMs.

In the near future, an implementation of the maps into SixTrack is planned, and we expect to see a significant speed up of the current computations. When using a higher-order expansion of the GF, we expect to obtain an even better convergence with respect to the slice number, but at the same time the amount of CPU time required to find the roots of the implicit equation (27b) must be taken into consideration.

Finally, we emphasize that the method presented in Subsec. II C works for arbitrary, even time-dependent, Hamiltonians, where it might be difficult or impossible to find an explicit transformation map describing the complete operation.

\section{ACKNOWLEDGMENTS}

We would like to thank Frank Schmidt for the introduction to the problem, his enduring support and help with the MAD-X code as well as Irina Shreyber, Riccardo de Maria and Laurent Deniau for fruitful discussions. We also thank the sponsoring by the "Wolfgang Gentner Programm" of the German Federal Ministry of Education and Research (BMBF).

\section{APPENDIX A: ON THE HAMILTONIAN IN FRENET-SERRET COORDINATES}

Let us begin with the Hamiltonian describing the motion of a charged particle in an electromagnetic field, formulated in cartesian coordinates. This Hamiltonian can be found for 
example in Ref. [12], p. 24 Eq. (1.92) or in Ref. [18], p. 351 Eq. (8.56) (here we are using SI units)

$$
\mathcal{H}=c \sqrt{(\vec{p}-e \vec{A})^{2}+m^{2} c^{2}}+e \varphi .
$$

where $\vec{p}=\left(p_{x}, p_{y}, p_{z}\right)$ denotes the canonical momenta of the particle.

In the situation of circular accelerators it is often more convenient to change the cartesian coordinate system, in which the Hamiltonian (A1) is formulated, towards a comoving coordinate system with respect to a reference trajectory. In the original Cartesian coordinates let us denote this reference trajectory by $\vec{r}(s)=(x(s)$, $y(s), z(s))$. We will assume that it is parametrized by arc length, i.e. $\vec{t}(s):=\frac{d}{d s} \vec{r}(s)$ has norm $|\vec{t}(s)|=1$ for all $s$.

Let $\vec{n}(s), \vec{b}(s)$ be unit vectors which are smoothly dependent on $s$ and so that $(\vec{t}, \vec{n}, \vec{b})$ constitute a righthanded-oriented orthonormal basis. For example, if $\vec{t}(s)$ and $\frac{d}{d s} \vec{t}(s)$ are linearly independent for every $s$, one can choose $\vec{n}$ to be the normalized $-\frac{d}{d s} \vec{n}$ and take the cross product $\vec{t} \times \vec{n}$ to define $\vec{b}$.

Assume that the map $f:(\xi, \eta, s) \mapsto \vec{r}(s)+\xi \vec{n}(s)+$ $\eta \vec{b}(s)=(x, y, z)$ is injective on an open domain $U$ containing a curve at $\xi=0=\eta$, i.e. it provides a map around a section of the reference trajectory. We call the map $f$ a Frenet-Serret coordinate system. Let us denote the tuple of new coordinates $(\xi, \eta, s)$ with $Q$ and consider the realvalued function $F_{3}$, given by

$$
F_{3}(Q, p):=-\vec{p}(\vec{r}(s)+\xi \vec{n}(s)+\eta \vec{b}(s)) .
$$

Remark A.1. $-F_{3}$ is a GF of type 3.

Proof.-

(i) For the spatial coordinates $x, y$ and $z$ we have the (local) bijection

$$
(x, y, z)=-\frac{\partial F_{3}}{\partial \vec{p}}=f(\xi, \eta, s) .
$$

(ii) The map $F_{3}$ is, by construction, differentiable with respect to the new coordinates $Q$. Let us denote the corresponding negative partial derivatives of $F_{3}$ with respect to these coordinates by $p_{\xi}, p_{\eta}$ and $p_{s}$ respectively. For $p_{\xi}$ and $p_{\eta}$ we have

$$
p_{\xi}:=-\frac{\partial F_{3}}{\partial \xi}=\vec{p} \vec{n}, \quad p_{\eta}:=-\frac{\partial F_{3}}{\partial \eta}=\vec{p} \vec{b} .
$$

The expression for $p_{s}$ is slightly more complicated. Since $\frac{d}{d s} \vec{n} \perp \vec{n}$ and similarly for the other vectors, we can deduce that there must exist differentiable functions $\alpha_{1}, \alpha_{2}$ and $\alpha_{3}$, which are in general dependent on $s$, with

$$
\begin{gathered}
\frac{d}{d s} \vec{t}=\alpha_{1} \vec{n}+\alpha_{2} \vec{b}, \\
\frac{d}{d s} \vec{n}=-\alpha_{1} \vec{t}+\alpha_{3} \vec{b}, \\
\frac{d}{d s} \vec{b}=-\alpha_{2} \vec{t}-\alpha_{3} \vec{n} .
\end{gathered}
$$

(If $\alpha_{3}$ is zero we say that the comoving frame is torsion free. It is not hard to see in this case that if $\alpha_{1}$ or $\alpha_{2}$ is also zero, then the reference curve must lay in a plane.) These functions are usually given by the reference trajectory itself (see above) and therefore are assumed to be known. With Eqs. (A4a)-(A4c) we get

$$
\begin{aligned}
p_{s} & :=-\frac{\partial F_{3}}{\partial s}=\vec{p} \vec{t}+\xi \vec{p} \frac{d}{d s} \vec{n}+\eta \vec{p} \frac{d}{d s} \vec{b} \\
& =\left(1-\alpha_{1} \xi-\alpha_{2} \eta\right) \vec{p} \vec{t}+\alpha_{3} \xi \vec{p} \vec{b}-\alpha_{3} \eta \vec{p} \vec{n} .
\end{aligned}
$$

By Eq. (A2) and our assumption on $f$, we have a local bijective relation between the spatial coordinates $x, y$ and $z$ and the new coordinates $\xi, \eta$ and $s$. Together with Eqs. (A3) and (A5) for the new momenta this proves that $F_{3}$ generates a local bijection between all new and old variables, while satisfying the equations for a GF of type 3. This means that it generates a canonical transformation between the cartesian- and the Frenet-Serret coordinates.

Before we proceed with the Hamiltonian, let us analyze the parametrization $f$ a bit further. Again using (A4a)(A4c), and taking the exterior differential componentwise, we get

$$
\begin{aligned}
(d x, d y, d z)=d f= & \left(\left(1-\alpha_{1} \xi-\alpha_{2} \eta\right) \vec{t}+\alpha_{3} \xi \vec{b}\right. \\
& \left.-\alpha_{3} \eta \vec{n}\right) d s+\vec{n} d \xi+\vec{b} d \eta .
\end{aligned}
$$

With the standard metric in $\mathbb{R}^{3}, g=d x^{2}+d y^{2}+d z^{2}$, we therefore obtain in the new coordinates

$$
\begin{aligned}
d l^{2}:= & f^{*} g=\left[\left(1-\alpha_{1} \xi-\alpha_{2} \eta\right)^{2}+\alpha_{3}^{2} \xi^{2}+\alpha_{3}^{2} \eta^{2}\right] d s^{2} \\
& -\alpha_{3} \eta(d s d \xi+d \xi d s)+\alpha_{3} \xi(d s d \eta+d \eta d s) \\
& +d \xi^{2}+d \eta^{2} .
\end{aligned}
$$

From now on we will assume that the given Frenet-Serret system is torsion free, i.e. describes an orthogonal curvilinear coordinate system with metric

$$
d l^{2}=\left(1-\alpha_{1} \xi-\alpha_{2} \eta\right)^{2} d s^{2}+d \xi^{2}+d \eta^{2} .
$$


Let us recall the coordinate-independent definition of the curl of a vector field $\vec{X}$. If $\vec{m}$ is an arbitrary vector, the $\vec{m}$-component of the curl $\vec{\nabla} \times \vec{X}$ is given by

$$
(\vec{\nabla} \times \vec{X}) \cdot \vec{m}:=\lim _{A \rightarrow 0} \frac{1}{|A|} \oint_{\partial A} \vec{X} d \vec{r}
$$

where the integration goes along the boundary of a surface $A$ of area $|A|$ which is orthogonal to $\vec{m}$ (orthogonal with respect to the standard scalar product). The vectors $\vec{t}, \vec{n}$ and $\vec{b}$ constitute an orthonormal basis of our Frenet-Serret system: They are given as the normalized vectors of the push-forward of the basis vectors $\partial_{s}, \partial_{\xi}$ and $\partial_{\eta}$ via $f$, i.e. by $d f\left(\partial_{s}\right)=\partial_{s} f, d f\left(\partial_{\xi}\right)=\partial_{\xi} f$ and $d f\left(\partial_{\eta}\right)=\partial_{\eta} f$ and normalization. By inserting for $\vec{m}$ in Eq. (A8) this basis, the curvilinear version of the curl becomes (see Ref. [19])

$$
\begin{gathered}
(\vec{\nabla} \times \vec{X})_{t}=\partial_{\xi} X_{b}-\partial_{\eta} X_{n}, \\
(\vec{\nabla} \times \vec{X})_{n}=\frac{1}{1-\alpha_{1} \xi-\alpha_{2} \eta}\left[\partial_{\eta}\left(\left(1-\alpha_{1} \xi-\alpha_{2} \eta\right) X_{t}\right)-\partial_{s} X_{b}\right], \\
(\vec{\nabla} \times \vec{X})_{b}=\frac{1}{1-\alpha_{1} \xi-\alpha_{2} \eta}\left[\partial_{s} X_{n}-\partial_{\xi}\left(\left(1-\alpha_{1} \xi-\alpha_{2} \eta\right) X_{t}\right)\right] .
\end{gathered}
$$

It is important to note that, by using the coordinateindependent formulation (A8), all components emerging in Eqs. (A9a)-(A9c) are taken with respect to the comoving basis $\vec{t}, \vec{n}$ and $\vec{b}$ respectively, i.e. with respect to the representation $\vec{X}=X_{t} \vec{t}+X_{n} \vec{n}+X_{b} \vec{b}$.

Remark A.2.-The curl formula does not hold for the components of the local representation $\vec{X}=X_{s} \partial_{s}+$ $X_{\xi} \partial_{\xi}+X_{\eta} \partial_{\eta}$ of $\vec{X}$ in terms of the coordinates $s, \xi$ and $\eta$; these components are related to the previous representation via the push-forward with $f$ :

$$
\begin{aligned}
f^{\prime}(\vec{X}) & =\left(\partial_{\xi} f, \partial_{\eta} f, \partial_{s} f\right)(X)=X_{s} \partial_{s} f+X_{\xi} \partial_{\xi} f+X_{\eta} \partial_{\eta} f \\
& =\left(1-\alpha_{1} \xi-\alpha_{2} \eta\right) X_{s} \vec{t}+X_{\xi} \vec{n}+X_{\eta} \vec{b},
\end{aligned}
$$

i.e.

$$
\begin{aligned}
& X_{t}=\left(1-\alpha_{1} \xi-\alpha_{2} \eta\right) X_{s}, \\
& X_{n}=X_{\xi}, \\
& X_{b}=X_{\eta} .
\end{aligned}
$$

Let us now turn our attention to the Hamiltonian (A1). Since $F_{3}$ is independent of the time, the new Hamiltonian, which we shall denote by $\mathcal{K}$, is related to $\mathcal{H}$ by expressing the old coordinates in terms of the new coordinates: $\mathcal{K}(Q, P)=\mathcal{H}(q, p)$ with $P:=\left(p_{\xi}, p_{\eta}, p_{s}\right), q:=(x, y, z)=$ $q(Q, P)$ and $p=p(Q, P)$. Note that the expression $(\vec{p}-e \vec{A})^{2}$ is invariant against orthogonal transformations in momentum space

$$
(\vec{p}-e \vec{A})^{2}=(\vec{p} \vec{t}-e \vec{A} \vec{t})^{2}+(\vec{p} \vec{n}-e \vec{A} \vec{n})^{2}+(\vec{p} \vec{b}-e \vec{A} \vec{b})^{2} .
$$

Using Eqs. (A3), (A5), (A11b) and (A11c) we then conclude for the Hamiltonian in the new coordinates

$$
\begin{aligned}
\mathcal{K} & \left.=c\left(\left(1-\alpha_{1} \xi-\alpha_{2} \eta\right)^{-1} p_{s}-e A_{t}\right)^{2}+\left(p_{\xi}-e A_{\xi}\right)^{2}+\left(p_{\eta}-e A_{\eta}\right)^{2}+m^{2} c^{2}\right)^{1 / 2}+e \varphi \\
& \Rightarrow p_{s}=\left(1-\alpha_{1} \xi-\alpha_{2} \eta\right) \sqrt{c^{-2}(\mathcal{K}-e \varphi)^{2}-\left(p_{\xi}-e A_{\xi}\right)^{2}-\left(p_{\eta}-e A_{\eta}\right)^{2}-m^{2} c^{2}}+\left(1-\alpha_{1} \xi-\alpha_{2} \eta\right) e A_{t}
\end{aligned}
$$

where we have left $A_{t}$ in its form, since we will make use of the curl formulas (A9a)-(A9c) later on anyways, in which $A_{t}$ is of importance instead of $A_{s}$. The potential functions are taken at the point $(x, y, z)=f(\xi, \eta, s)$. With Eqs. (A12) and (A13) we have made two steps: We have obtained a Hamiltonian (A12) in terms of Frenet-Serret coordinates out of the Hamiltonian (A1) formulated in Cartesian coordinates. And we have rearranged the expression toward $p_{s}$, which will later play the role as the negative of a new Hamiltonian in which the (negative) energy and time play the role as two conjugate generalized coordinates.
At this point let us list a few references where the form of the Hamiltonian (A12) is derived. The Hamiltonian (A12) can be found in Wolski's book by a different approach [20], p. 74 Eq. (2.72), and in Wiedemann's book [12], p. 29 Eq. (1.116). In Ripken's paper [6], p. 10 Eq. (2.21) the final form of Eq. (A13) can be found. Wiedemann's derivation differs only slightly than ours: In contrast to his derivation we used here a potential-independent GF in order to have a clear understanding what role the coefficients of the vector potential play with respect to the embedding $f$ and the curl formulas (A9a)(A9c) in curvilinear orthogonal coordinates. 
The change from $\mathcal{K}$ to $-p_{s}$ as the new Hamiltonian is left to the reader; we just mention at this point that this basically stems from the fact that the 2-Form $\omega$, which leads to the Hamilton-equations $l_{X} \omega=0, X(t) \equiv 1$, has the form

$$
\omega=d p_{\xi} \wedge d \xi+d p_{\eta} \wedge d \eta+d p_{s} \wedge d s-d \mathcal{K} \wedge d t .
$$

Note that apart of this subsection we have adopted a more common notation for the rest of this paper: $\xi \rightarrow x, \eta \rightarrow y$, $-\alpha_{1} \rightarrow K_{x},-\alpha_{2} \rightarrow K_{y}$.

\section{APPENDIX B: SUPPLEMENTAL MATERIAL}

Let us denote with $P_{k} \subset P_{\mathbb{C}}[x, y]$ the set of complex homogeneous polynomials of degree $k$. We will show a small Lemma:

Lemma B.1: Let $\nu: P_{1} \rightarrow P_{1}$ be a complex isomorphism. Then this map induces a bijection $\nu^{*}$ on the entire $P_{\mathbb{C}}[x, y]$ via $\nu^{*} g(x, y):=g(\nu(x), \nu(y))$.

Proof.-By construction, $\nu^{*}\left(P_{k}\right) \subset P_{k}$. Therefore it is sufficient to restrict to those kind of polynomials. Surjectivity: If $g \in P_{k}$ is given, then $\tilde{g}$ with $\tilde{g}(x, y):=$ $g\left(\nu^{-1}(x), \nu^{-1}(y)\right)$ is a polynomial in $P_{k}$ with $\nu^{*}(\tilde{g})=g$. Injectivity: Denote $r_{+}:=\nu(x)=: \nu_{11} x+\nu_{21} y, r_{-}:=\nu(y)=$ : $\nu_{12} x+\nu_{22} y$ and let $g_{j} \in \mathbb{C}$ for $j=0, \ldots, k$. Then the following holds for all $k \geq 0$ :

$$
0=\sum_{j=0}^{k} g_{j} r_{+}^{k-j} r_{-}^{j} \Rightarrow \forall j: g_{j}=0 .
$$

This assertion can be proven by induction over $k$. The case $k=0$ is clear. If $k=1$, then we have

$$
\begin{aligned}
0=g_{0} r_{+}+g_{1} r_{-} & =\left(\nu_{11} x+\nu_{21} y, \nu_{12} x+\nu_{22} y\right)\left(\begin{array}{l}
g_{0} \\
g_{1}
\end{array}\right) \\
& =(x, y)\left(\begin{array}{ll}
\nu_{11} & \nu_{12} \\
\nu_{21} & \nu_{22}
\end{array}\right)\left(\begin{array}{l}
g_{0} \\
g_{1}
\end{array}\right) .
\end{aligned}
$$

Since this equation holds for all $x$ and $y$, it follows that $(\nu)_{2 \times 2}\left(g_{0}, g_{1}\right)^{t r}=0$, thus $g_{0}=0=g_{1}$. Note that this property holds even if $g_{0}$ and $g_{1}$ are polynomials. Now assume that the claim holds for some $k-1$ with $k \geq 1$. Then

$$
\sum_{j=0}^{k} g_{j} r_{+}^{k-j} r_{-}^{j}=\left(\sum_{j=0}^{k-1} g_{j} r_{+}^{k-1-j} r_{-}^{j}\right) r_{+}+g_{k} r_{-}^{k-1} r_{-}
$$

By the case for $k=1$ we have $\sum_{j=0}^{k-1} g_{j} r_{+}^{k-1-j} r_{-}^{j}=0$ and $g_{k} r_{-}^{k-1}=0$, thus $g_{k}=0$ and by assumption $\forall j \in$ $\{0, \ldots, k-1\}: g_{j}=0$. This proves the claim above. Since $\nu^{*}$ is linear by definition, the claim implies that $\nu^{*}$ is injective.

\section{APPENDIX C: NOTATIONS IN MAD-X}

The program MAD-X uses the following notation [21] (For details regarding orbit (5) see Appendix D):

$$
\begin{aligned}
\operatorname{orbit}(1) & =x, \\
\operatorname{orbit}(2) & =p_{x} \\
\operatorname{orbit}(3) & =y \\
\operatorname{orbit}(4) & =p_{y} \\
\operatorname{orbit}(5) & =\beta_{0}^{-1} \sigma, \\
\operatorname{orbit}(6) & =\frac{\Delta E}{p_{0} c}=\frac{\Delta E}{\beta_{0} E_{0}}=\frac{1}{\beta_{0}} \eta=\beta_{0} p_{\sigma}, \\
\operatorname{deltap} & =\frac{\Delta p}{p_{0}}=\hat{\eta}, \\
\operatorname{elrad} & =\Delta s \\
\operatorname{dipr} & =K_{x} \Delta s \\
\operatorname{dipi} & =K_{y} \Delta s
\end{aligned}
$$

We note that the parameter deltap in MAD-X is used in case of particle tracking as a global correction for the orbit(6) components of the particles, via Eq. (17).

\section{APPENDIX D: orbit(5) CONVERSION}

We recall that

$$
\sigma=s-v_{0} t
$$

The coordinate orbit (5) corresponds to $-c$ times the difference of the particle time minus the time of the reference particle [21]. A positive orbit (5) means that the particle arrives ahead of the reference particle. This definition has to be taken with respect to a given position in the ring, which makes the conversion more involved: As above, at time $t$, let the particle of question be at position $s$. Let us say that at time $t^{\prime}<t$ the reference particle was at $s^{\prime}<s$ and the reference particle crosses $s$ at time $t_{r}>t^{\prime}$. So during the time $t_{r}-t^{\prime}$ the reference particle travels the distance $v_{0}\left(t_{r}-t^{\prime}\right)$, which must be equal to $s-s^{\prime}$, so $t_{r}=t^{\prime}+\left(s-s^{\prime}\right) / v_{0}$. From the definition we get

$$
\begin{aligned}
\operatorname{arbit}(5)= & -c\left(t-t_{r}\right)=c\left(t^{\prime}-t\right)+\beta_{0}^{-1}\left(s-s^{\prime}\right) \\
= & -c t+\beta_{0}^{-1} s+c t^{\prime}-\beta_{0}^{-1} s^{\prime}=\beta_{0}^{-1} \sigma \\
& +c\left(t^{\prime}-s^{\prime} / v_{0}\right) .
\end{aligned}
$$

According to Ref. [21] p. 12, the values $\left(t^{\prime}, s^{\prime}\right)$ are set to $(0,0)$ unless otherwise specified by the user. In this case, $t_{r}=s / v_{0}$ and orbit(5) $=\beta_{0}^{-1} \sigma$. These user-dependent 
values must be included in the orbit transformations if this feature is required. Fortunately, this problem does not play a role for derivatives, since the constants drop out.

\section{APPENDIX E: CODE MODIFICATIONS}

We have done code modifications for MAD-X v.5.02.07 on two occasions, frequently after the statement if (elrad.gt.zero.and.get_option(' thin foc' ) .eq.1), which tells the program to use our new code for CFMs after makethin. The modifications presented here may not yet be fully optimized, but should be sufficient for our purposes.

1. For twiss we modified in twiss.f90 the subroutine tmmult. We changed the orbit, re and te computations, after undoing the previous changes in these entries, as follows: (i) For the orbit computations we are using the kick map given in Subsection III B:

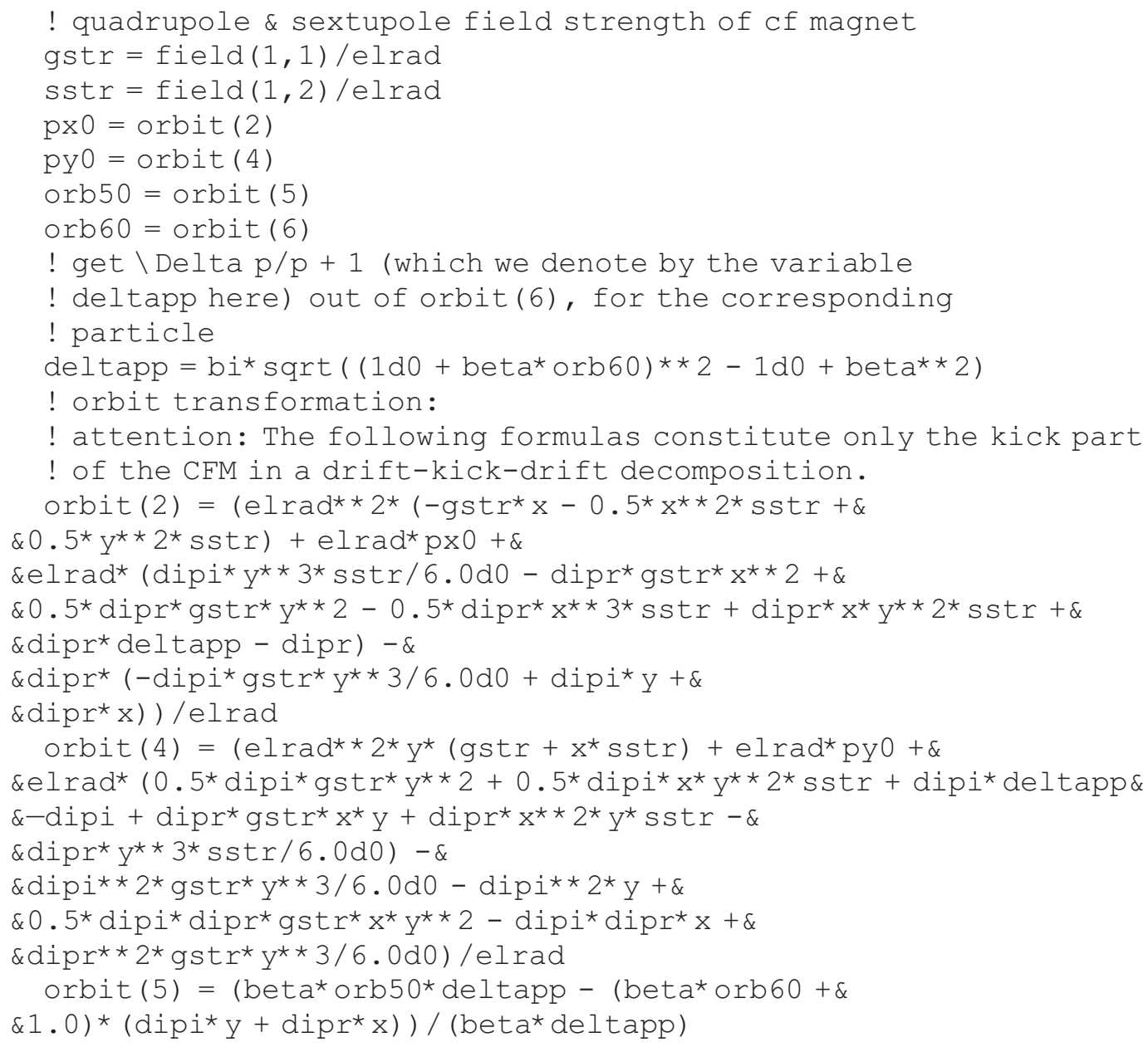

where $\mathrm{x}=\operatorname{orbit}(1)$ and $\mathrm{y}=\operatorname{orbit}(3)$. We have taken care of the deltap-handling in MAD-X by recomputing $\Delta p / p_{0}$ for the corresponding particle out of its orbit (6) component.

(ii) For re (and later te), this kick map cannot be used, since it does not change the transverse coordinates (it does not represent the entire transformation), especially the derivatives of these coordinates with respect to the other coordinates can not be obtained in this way. However $r e$ and te are the coefficients of the expansion at zero of the full transformation, modified by the inverses of the drift parts, so the transformation described in Subsec. III C, which is symplectic at zero, is sufficient for the computations. For re, the first derivative of this transformation yields 
! quadrupole field strength of cf magnet.

! the sextupole makes no contribution for 1st order

! gstr is defined here without elrad-factor, since this factor drops

! out again in the re-formulas.

gstr $=$ field $(1,1)$

re $(2,1)=-$ dipr**2/elrad - gstr

re $(2,3)=-$ dipi*dipr/elrad

re $(2,6)=$ dipr/beta

re $(4,1)=r e(2,3)$

re $(4,3)=-$ dipi**2/elrad + gstr

re $(4,6)=$ dipi/beta

re $(5,1)=-r e(2,6)$

$r e(5,3)=-r e(4,6)$

(iii) For te, the second derivative (with $1 / 2$ factor) yields

! quadrupole, sextupole, ... field strength of cf magnet

! to avoid some operations which appear frequently,

! we define these strengths a bit differently (as in te)

gstr $=$ field $(1,1) /$ elrad

sstr $=$ field $(1,2) / 2.0$

te $(1,1,2)=0.5 *$ dipr

te $(1,2,1)=\operatorname{te}(1,1,2)$

te $(1,2,3)=0.5 *$ dipi

te $(1,3,2)=\operatorname{te}(1,2,3)$

te $(2,1,1)=-$ dipr*gstr-sstr

te $(2,2,2)=-\operatorname{te}(1,1,2)$

$\operatorname{te}(2,3,3)=\operatorname{te}(1,1,2) * \operatorname{gstr}+\operatorname{sstr}$

$\operatorname{te}(2,4,4)=-\operatorname{te}(1,1,2)$

$\operatorname{te}(2,6,6)=\operatorname{te}(1,1,2)-\operatorname{te}(1,1,2) /$ beta**2

$\operatorname{te}(3,1,4)=\operatorname{te}(1,1,2)$

$\operatorname{te}(3,3,4)=\operatorname{te}(1,2,3)$

$\operatorname{te}(3,4,1)=\operatorname{te}(1,1,2)$

$\operatorname{te}(3,4,3)=\operatorname{te}(1,2,3)$

$\operatorname{te}(4,1,3)=\operatorname{te}(2,3,3)$

te $(4,2,2)=-\operatorname{te}(1,2,3)$

$\operatorname{te}(4,3,1)=\operatorname{te}(2,3,3)$

$\operatorname{te}(4,3,3)=\operatorname{te}(1,2,3) * \operatorname{gstr}$

te $(4,4,4)=-\operatorname{te}(1,2,3)$

$\operatorname{te}(4,6,6)=\operatorname{te}(1,2,3)-\operatorname{te}(1,2,3) /$ beta**2

te $(5,1,6)=-\operatorname{te}(2,6,6)$

$\operatorname{te}(5,3,6)=-\operatorname{te}(4,6,6)$

$\operatorname{te}(5,6,1)=-\operatorname{te}(2,6,6)$

te $(5,6,3)=-\operatorname{te}(4,6,6)$

2. For track we have modified in trrun. $f 90$ the subroutine ttmult as follows:

(i) We have commented out any previous operation in case of nord $>0 \wedge$ elrad $>0 \wedge$ thinfoc $=1$.

(ii) We have added the following if-statement before the tracking computations, which is meant as a temporary solution: if (elrad.le.0d0.or.get_option('thin_foc' ).ne.1) then!

(iii) In case the above if-statement is not fulfille $\bar{d}$, i.e. we are in the case of a thin CFM, we have added in the corresponding else-statement the same kick map as in the case of the orbit computations in twiss. f90:

! cf magnet with quadrupole \& sextupole

gstr $=$ field $(1,1) /$ elrad

sstr $=$ field $(1,2) /$ elrad

do jtrk $=1$, ktrack

$\mathrm{x}=\operatorname{track}(1, j \operatorname{trk})$

$\mathrm{px} 0=\operatorname{track}(2, j \mathrm{trk})$

$y=\operatorname{track}(3, j \operatorname{trk})$

pyo $=\operatorname{track}(4, j$ trk $)$ 


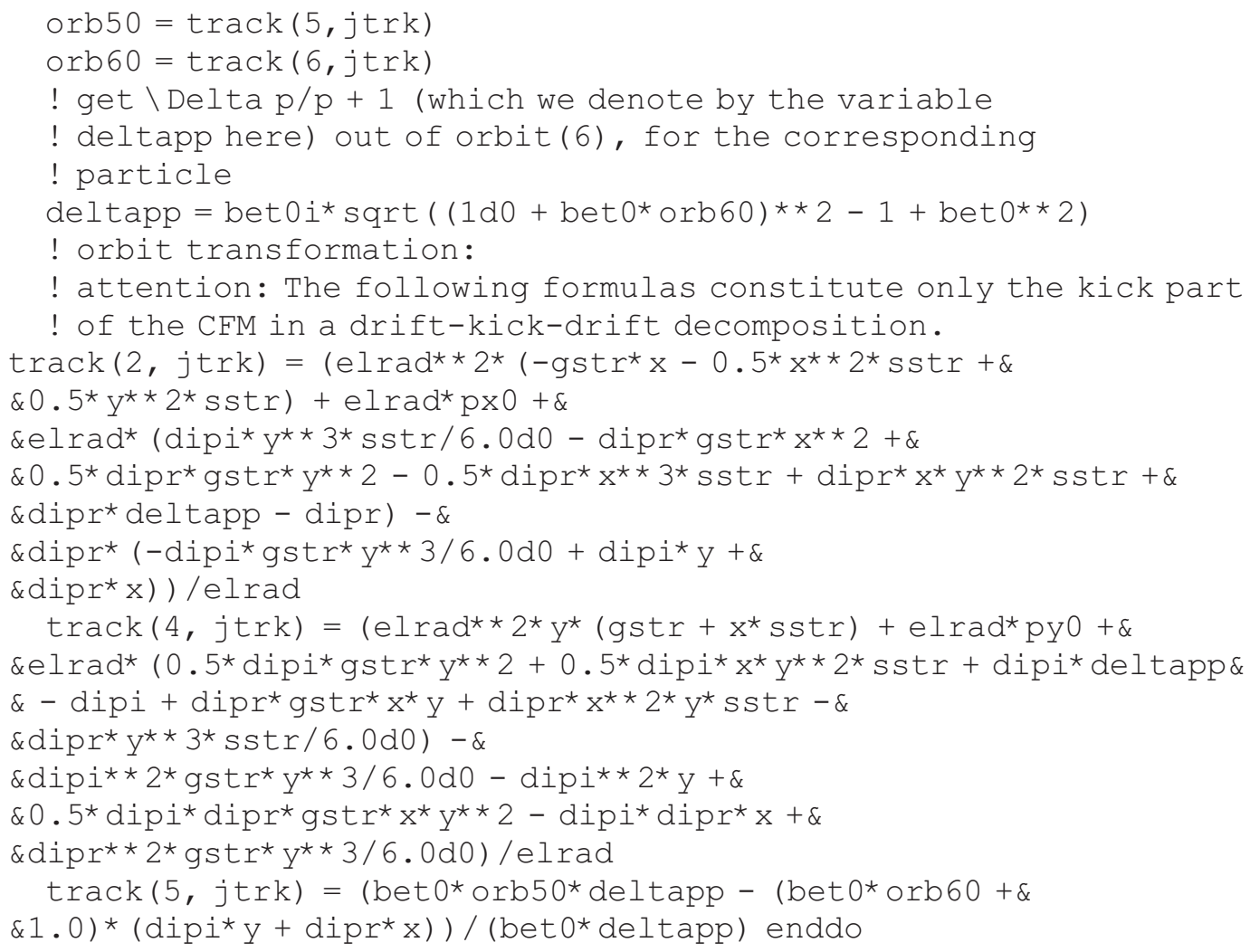

As in the case of the orbit computations, we have computed $\Delta p / p_{0}$ for every particle number jtrk.

The code to compute the potential for various magnet setups based on the considerations of this article, to generate the corresponding FORTRAN code and to perform basic symplecticity checks was written in PYTHON (in form of an interactive Jupyter notebook). The latest version of the used MAD-X files and the IPython notebooks can be found in Ref. [5].

[1] E. Forest, Beam Dynamics, Physics and Technology of Particle and Photon Beams Vol. 8 (Taylor \& Francis, London, 1998).

[2] K. L. Brown, SLAC Research Report No. 75, Stanford University, California, 1982.

[3] S. Fartoukh, Ph.D. thesis, Universite Paris VI, 1997.

[4] F. C. Iselin, Lie transformations and transport equations for combined-function dipoles, Particle Accelerators 17, 143 (1985).

[5] M. Titze, https://github.com/mtitze/madx-cf.

[6] G. Ripken, DESY Technical Report No. 85-084, 1985.

[7] D. P. Barber, G. Ripken, and F. Schmidt, DESY Technical Report No. 87-036, 1987.

[8] P. J. Channell, Los Alamos National Laboratory Technical Report No. AT-6:ATN-83-9, 1983.

[9] R. D. Ruth, A canonical integration technique, Trans. Nucl. Sci. NS-30, 2669 (1983).
[10] P. J. Channell and C. Scovel, Symplectic integration of Hamiltonian systems, Nonlinearity 3, 231 (1990).

[11] K. Feng and M. Qin, Symplectic Geometric Algorithms for Hamiltonian Systems (Springer, Berlin Heidelberg, 2010).

[12] H. Wiedemann, Particle Accelerator Physics, 3rd ed. (Springer, Berlin Heidelberg, 2007).

[13] D. P. Barber, K. Heinemann, G. Ripken, and F. Schmidt, DESY Technical Report No. 96-156, 1996.

[14] SymPy Development Team, SymPy: Python library for symbolic mathematics, 2016.

[15] E. Jones, T. Oliphant, P. Peterson et al., SciPy: Open source scientific tools for Python, 2001-. Online.

[16] S. van der Walt, S. C. Colbert, and G. Varoquaux, The numpy array: A structure for efficient numerical computation, Comput. Sci. Eng. 13, 22 (2011).

[17] J. D. Hunter, Matplotlib: A 2d graphics environment, Comput. Sci. Eng. 9, 90 (2007).

[18] H. Goldstein, C. P. Poole, and J. L. Safko, Classical Mechanics (Addison Wesley, Reading, MA, 2002).

[19] J. Foadi, Gradient, Divergence and Curl in Curvilinear Coordinates, 2007; http://www.jfoadi.me.uk/documents/ lecture_mathphys2_05.pdf.

[20] A. Wolski, Beam Dynamics in High Energy Particle Accelerators (Imperial College Press, London, 2014).

[21] H. Grote, F. Schmidt, and L. Deniau, The MAD-X Program: User's Reference Manual, Geneva, Switzerland, 2014 (CERN, Geneva, 2016); http://cern.ch/madx/releases/ last-dev/madxuguide.pdf. 